hep-th/0608027

CERN-PH-TH/2006-157

\title{
Effective superpotentials for B-branes in Landau-Ginzburg models
}

\author{
Suresh Govindarajan* ${ }^{*}$ and Hans Jockers ${ }^{\dagger}$ \\ * Institut für Theoretische Physik \\ ETH, Zürich, Switzerland \\ * Department of Physics, Indian Institute of Technology Madras \\ Chennai, India \\ ${ }^{\dagger}$ Department of Physics, Theory Division \\ CERN, Geneva, Switzerland
}

\begin{abstract}
We compute the partition function for the topological Landau-Ginzburg B-model on the disk. This is done by treating the worldsheet superpotential perturbatively. We argue that this partition function as a function of bulk and boundary perturbations may be identified with the effective $D$-brane superpotential in the target spacetime. We point out the relationship of this approach to matrix factorizations. Using these methods, we prove a conjecture for the effective superpotential of Herbst, Lazaroiu and Lerche for the $A$-type minimal models. We also consider the Landau-Ginzburg theory of the cubic torus where we show that the effective superpotential, given by the partition function, is consistent with the one obtained by summing up disk instantons in the mirror A-model. This is done by explicitly constructing the open-string mirror map.
\end{abstract}

August, 2006 


\section{Introduction}

Since their discovery, $D$-branes have played an important rôle in many branches of string theory. On the one hand $D$-branes appear as ingredients in semi-realistic string model building in both particle physics and more recently also in cosmology. On the other hand $D$-branes have shed light on the structure of string theory from various angles. For instance they have been crucial in revealing string dualities and have given us some insights into non-perturbative aspects of string theory.

Although there has been much progress over the years we still have a rather limited understanding of $D$-branes. Just to name one example, in the context of Calabi-Yau compactifications many aspects of $D$-branes are only explored at the large volume point in the Kähler moduli space. In this regime quantum corrections are suppressed and a description in terms of classical geometry is applicable, whereas very little is known at generic points in the Kähler moduli space. However there exists another special point in the non-geometric region of the Kähler moduli space in terms of Landau-Ginzburg orbifolds. These theories flow in the infrared to conformal field theories (CFTs), and for particular subclasses of Landau-Ginzburg orbifolds (which usually correspond to special points in the complex structure moduli space) these CFTs at the IR fixed point are known to be given by appropriate Gepner models [1,2]. The physics of $D$-branes in the context of Gepner models has been investigated extensively, whereas only recently progress has been made in studying $D$-branes in the broader context of Landau-Ginzburg theories [3, 4, 5, 4, 4, 6, 4, 8].

Already the two-dimensional bulk Landau-Ginzburg models with $(2,2)$ supersymmetry have proven useful in providing a computational framework for CFTs and their perturbations by relevant as well as marginal operators. They allow us to use free-field theory methods to extract information at the CFT end. Examples include

the computation of the central charge, the Ramond characters of minimal models and an off-shell description of the superconformal algebra [9].

The inclusion of $D$-branes in Landau-Ginzburg models leads one to consider Landau-Ginzburg models with boundaries. These theories are richer in content due to the reduced amount of supersymmetry, however, they are also more difficult. In order to deal with this increased complexity new tools such as the boundary linear $\sigma$-models [10, 11, 12, 3], and matrix factorizations have been developed [4, 5, 4, 6, 6, 7, 8]. 
A further understanding of the successes and limitations of Landau-Ginzburg models is obtained from studying their topological twisted versions. Using this approach one describes only a subsector of the original physical string theory. This, however, also becomes a virtue as this subsector is decoupled from all Kähler moduli, and as a consequence the topological Landau-Ginzburg models describe quantities, which have an invariant meaning in the whole Kähler moduli space. In the lowenergy effective description of the underlying physical string theory these invariants can often be identified with holomorphic quantities protected by spacetime supersymmetry [13, 14, 15, 16, 17]. Prominent examples are prepotentials in $N=2$ theories [13, 14] and gauge kinetic coupling functions and effective superpotentials in $N=1$ theories [13, 15, 16, 18, 19, 17].

The purpose of this paper is to present a technique to compute effective $D$ brane superpotentials by taking advantage of the computational framework provided by (topological) Landau-Ginzburg theories. One way to compute effective $D$-brane superpotentials in Landau-Ginzburg theories is to study obstructions to matrix factorizations [20,21, 8,22]. Then these obstructions can be integrated to an effective $D$ brane superpotential. Although there is a recursive algorithm in doing so [23, 24, 22], for more involved examples the procedure can become cumbersome. Here we provide for an alternative approach by computing directly the effective $D$-brane superpotential perturbatively. The key idea is to view the Landau-Ginzburg model as a free theory, where the Landau-Ginzburg superpotential is treated as mere perturbation [25]. From this perspective the effective $D$-brane superpotential is simply computed by summing appropriate Feynman diagrams of the free theory. This technique is somewhat orthogonal to the methods discussed in the context of matrix factorizations. However, ultimately both approaches are equivalent [26,27], as we will also anticipate here.

We perform our explicit computations for the Landau-Ginzburg models of the $A$-type minimal models and for the Landau-Ginzburg model associated to the twodimensional torus. However, most of the presented analysis is much more general and applies also for Landau-Ginzburg models of Calabi-Yau spaces as has already been demonstrated in ref. [25].

The outline of the paper is as follows. In section 2 we review Landau-Ginzburg theories without boundaries, which are capable to describe the closed string sector. 
Besides fixing the notation and introducing our two prime examples, the LandauGinzburg theory of the $A$-type minimal model and of the torus, we already emphasis certain aspects which become important later in the context of the perturbative treatment of the Landau-Ginzburg superpotential.

The addition of boundaries to $(2,2)$ supersymmetric Landau-Ginzburg models is discussed in section 3 . Here we also show the relation between matrix factorizations and the boundary conditions which we impose for our computation. Finally, we come back to our two examples for which we describe the $D$-brane configurations considered in the forthcoming analysis.

In section 4 we argue that the topological disk partition function of LandauGinzburg models computes effective $D$-brane superpotentials. This is demonstrated by explicitly computing the topological disk partition function for the $A$-type minimal model at level $k$. We find precise agreement with the effective $D$-brane superpotentials computed for low levels of $k$ in refs. [28,29]. Our general result confirms also the conjecture of refs. 28,29] for the general structure of the effective superpotential in the context of the $A$-minimal model for higher values of $k$.

In section 5 we apply the perturbative computation to the torus. This example is much more involved due to the fact that the $D$-brane spectrum contains now a marginal operator. As a consequence the effective $D$-brane superpotential becomes an infinite series in terms of the modulus associated to this marginal open-string operator. We determine general features of the $D$-brane superpotential and we develop the tools to explicitly compute the first few terms in this infinite series.

In section 6 we use for the first time the modular properties of the torus in order to gain further insight into the structure of the effective $D$-brane superpotential for the torus example. This allows us to confirm certain symmetry properties of the effective $D$-brane superpotential already anticipated in the previous section.

For the two-dimensional torus there is a mirror description in the topological A-model on the mirror torus. Thus in section 7 we map $D$-brane configurations of the torus in the B-model to the mirror A-model along the lines of ref. [30]. Since the torus geometry is simple enough one is able to derive the A-model $D$-brane effective superpotential, which appears as a sum of disk instantons [30,31,32, 33]. By comparing the superpotentials on both sides, we are able to construct the open-string mirror 
map. Since the mirror map fulfills a set of over-constrained equations, we obtain a highly non-trivial check on our method of computing $D$-brane superpotentials in the topological B-model. We end this section by some speculations on non-holomorphic terms in the context of the topological B-model. These terms have a natural origin in the topological A-model.

In section 8 we present our conclusions and in four appendices we collect some technical details of various sections in the main text.

\section{Landau-Ginzburg Models}

In order to set the stage for the forthcoming analysis, we review the $(2,2)$ supersymmetric Landau-Ginzburg models for the two-dimensional worldsheet, $\Sigma$. In choosing a worldsheet, $\Sigma$, without boundaries we describe the bulk theory or in other words the closed-string sector of these models. A thorough introduction as well as the detailed notational conventions used in this work can be found in ref. [9] (also see Appendix A).

The two-dimensional Landau-Ginzburg models with $(2,2)$ supersymmetry are constructed from chiral superfields, $\Phi$, which satisfy the chirality constraint

$$
\bar{D}_{\alpha} \Phi=0, \quad \alpha=+,-
$$

Here $\bar{D}_{\alpha}$ denotes the $(2,2)$ superspace derivative. The component fields of the chiral superfield, $\Phi$, are comprised of the complex boson, $\phi$, the fermionic fields, $\xi$ and $\tau$, and the complex auxiliary field, $F$.

In superspace notation the most general renormalizable action on the worldsheet, $\Sigma$, with several superfields, $\Phi^{i}$, is given by

$$
S_{\text {bulk }}=\int_{\Sigma} d^{2} x \int d^{4} \theta K(\Phi, \bar{\Phi})-\left(\lambda \int_{\Sigma} d^{2} x \int d \theta^{+} d \theta^{-} W(\Phi)+\text { h.c. }\right)
$$

where $x$ are the even coordinates of the worldsheet, $\Sigma$, and $\left(\theta^{ \pm}, \bar{\theta}^{ \pm}\right)$are the odd coordinates of the $(2,2)$ superspace. Furthermore, the function, $K$, is the Kähler potential and the holomorphic function, $W$, is the superpotential. We have also 
introduced a (formal) complex coupling constant, $\lambda$, multiplying the superpotential term.

In the topological B-model, which is the main focus of this work, two of the four supercharges are twisted to scalar operators, $\bar{Q}_{ \pm}$, by the B-type twisting. Hence these scalar operators are globally defined on any closed worldsheet, $\Sigma$, and therefore they become the BRST operators, $\bar{Q}_{ \pm}$, of the topological B-model [34]. In the action (2.2) one finds that the first and third terms are BRST exact. Hence, the partition function of the Landau-Ginzburg model is expected to depend holomorphically on the coupling constants that appear in the superpotential, $W$, and to be independent of the specific choice of Kähler potential, $K$. For our computations we will make the simple choice $K=\sum \Phi^{i} \bar{\Phi}_{i}$. We will further assume that the superpotential is quasi-homogeneous, i.e. there exist rational numbers, $\alpha_{i}$, for every chiral field $\Phi^{i}$ such that for any $\lambda \in \mathbb{C}^{*}$

$$
W\left(\lambda^{\alpha_{i} / 2} \Phi^{i}\right)=\lambda W\left(\Phi^{i}\right)
$$

The quasi-homogeneity of the superpotential ensures that we can identify and track the left- and right-moving $R$-symmetries away from the IR fixed point of the LandauGinzburg model 1 Further the central charge, $\hat{c}$, of the CFT is given by

$$
\hat{c}=\sum_{i}\left(1-\alpha_{i}\right)
$$

In models with several chiral fields, $\Phi^{i}$, we will be interested in Landau-Ginzburg orbifolds. These are orbifolds of the above models with a projection onto states with integral $R$-charge (the 'Gepner projection'). For specific superpotentials, $W$, such models are known to flow in the infrared to the CFT associated with Gepner models which in turn correspond to special points in both the Kähler and the complex structure moduli space of Calabi-Yau compactifications [1, 2, 35, 36, 37].

The topological observables of the bulk theory of the B-model are in the cohomology of the BRST operators $\bar{Q}_{+}$and $\bar{Q}_{-}$. This cohomology is invariant under the following scaling of the superpotential:

$$
W \rightarrow \lambda W
$$

1 Since our main interest is on worldsheets with boundaries, we will focus on the unbroken $R$-symmetry which is the sum of the left- and right-moving $R$-charge. This $R$-charge is given by shifting the naive free-field charge assignment by $\alpha_{i}$. Thus, the $R$-charge of $\Phi^{i}$ equals $\alpha_{i}$. 
The quasi-homogeneity of the superpotential implies that such a scaling can be undone by rescaling the fields $\Phi^{i} \rightarrow \lambda^{-\alpha_{i} / 2} \Phi^{i}$. This modifies the Kähler potential, $K$, which is, however, an exact piece in the topological theory. Thus, the cohomology is independent of the scaling parameter, $\lambda$. However, it is possible that the limit, $\lambda \rightarrow 0$, may be singular. It can also be shown by studying the localization in the topological Landau-Ginzburg model that the parameter $\lambda$ can be identified with the renormalization scale with $\lambda \rightarrow 0$ being the UV limit and $\lambda \rightarrow \infty$ being the IR limit $[38,39]$.

The topological BRST operator localizes on the space of zero-modes and takes the following form [3].39]:

$$
\left.\mathcal{Q} \sim\left(\bar{Q}_{+}+\bar{Q}_{-}\right)\right|_{\text {zeromodes }} \sim \bar{\partial}+i_{\partial W}
$$

Here $\bar{\partial}$ is the Dolbeaut operator of the non-compact target space, $X$, of the action (2.2), while the operator, $i_{\partial W}$, acts upon sections of the graded space, $\wedge \bullet T X$. The definition of $i_{\partial W}$ is induced by its action on vector fields

$$
i_{\partial W}\left(v^{j} \partial_{j}\right)=-i v^{j} \partial_{j} W
$$

which naturally extends to a general sections of the graded space, $\wedge^{\bullet} T X$, on which the operator, $i_{\partial W}$, becomes the odd derivation appearing in the BRST operator (2.6). Since $\bar{\partial}^{2}=i_{\partial W}^{2}=0$ and $\bar{\partial} i_{\partial W}+i_{\partial W} \bar{\partial}=0$, the topological observables are given by the double cohomology of the two differentials, $\bar{\partial}$ and $i_{\partial W}$. This usually involves computing a spectral sequence whose second term is $E_{2}=H_{i_{\partial W}}\left(H_{\bar{\partial}}(X)\right)$. In some situations, e.g. when the space, $X$, is given by $X=\mathbb{C}^{n}$ and when the superpotential, $W$, is a polynomial in these $n$ variables, the cohomology of $\mathcal{Q}$ is equal to $E_{2}$, i.e. $E_{2}=$ $E_{\infty}$. In other words the dimension of the double cohomology is simply determined by the cohomology of $i_{\partial W}$ within the cohomology group $H_{\bar{\partial}}(X)[40]$. Then we can treat the Landau-Ginzburg superpotential, $W$, perturbatively at the level cohomology and, as we will verify in the discussed examples, also at the level of correlation functions.

2 Locally the sections of $\wedge^{\bullet} T X$ are obtained as wedge products of vector fields. 


\subsection{Example 1: The A-type minimal model}

In this paper we will consider two examples. The first one is the $A$-type minimal model at level $k$. The Landau-Ginzburg description consists of a single chiral superfield, $\Phi$, and the Landau-Ginzburg superpotential

$$
W_{A_{k}}=\frac{\Phi^{k+2}}{k+2}-\sum_{j=2}^{k+2} g_{j}(t) \Phi^{k+2-j} .
$$

The coupling constants $g_{j}(t)=t_{j}+\cdots$ parametrize relevant bulk deformations about the conformal point and are taken to be functions of the flat coordinates $t_{2}, \ldots, t_{k+2}$ [41]. The conformal point is given by $g_{j}=0$. It is useful to treat the coefficient, $1 /(k+2)$, of $\Phi^{k+2}$ as the coupling, $g_{0}$.

As a particular example consider the $k=3$ model for which the Landau-Ginzburg superpotential takes the form

$$
W_{A_{3}}=\frac{\Phi^{5}}{5}-t_{2} \Phi^{3}-t_{3} \Phi^{2}-\left(t_{4}-t_{2}^{2}\right) \Phi-\left(t_{5}-t_{2} t_{3}\right) .
$$

The $R$-charge assignments for the component fields of the chiral multiplet, $\Phi$, resulting from eq. (2.3) at the conformal point are summarized in Table 1.

\begin{tabular}{|c|c|c|c|c|}
\hline Field & $\phi$ & $\tau$ & $\xi$ & $F$ \\
\hline$R$-charge & $\alpha$ & $\alpha-1$ & $\alpha-1$ & $\alpha-2$ \\
\hline
\end{tabular}

Table 1. $R$-charges for the $A_{k}$-minimal model in terms of $\alpha=\frac{2}{k+2}$.

\subsection{Example 2: The cubic torus}

The second example is the Landau-Ginzburg orbifold that flows in the infrared to the CFT, which describes strings on the two-dimensional torus, $\mathcal{T}$, at the LandauGinzburg point of the Kähler moduli space. This Landau-Ginzburg model consists of three chiral superfields, $\Phi^{i}$, the cubic Landau-Ginzburg superpotential

$$
W=\sum_{i=1}^{3}\left(\Phi^{i}\right)^{3}-3 a \Phi^{1} \Phi^{2} \Phi^{3},
$$


and the $\mathbb{Z}_{3}$-orbifold action, $\Phi^{i} \rightarrow e^{2 \pi i / 3} \Phi^{i}$. The coupling, $a$, parametrizes the complex structure of the cubic torus, $\mathcal{T}$. At the Fermat point, i.e. for $a=0$, in the complex structure moduli space the Landau-Ginzburg orbifold flows in the infrared to the $1^{3}$ Gepner model, i.e. it flows to the CFT obtained form the tensor product of three $k=1 A$-type minimal models subject to the Gepner projection [1, 2]. Therefore, as summarized in Table 2, the $R$-charges of the fields in the chiral multiplets, $\Phi^{i}$, of the cubic torus, $\mathcal{T}$, coincide with the $R$-charges of chiral multiplet in the $A$-type minimal model at level $k=1$.

\begin{tabular}{|c|c|c|c|c|}
\hline Field & $\phi$ & $\tau$ & $\xi$ & $F$ \\
\hline$R$-charge & $\frac{2}{3}$ & $-\frac{1}{3}$ & $-\frac{1}{3}$ & $-\frac{4}{3}$ \\
\hline
\end{tabular}

Table 2. R-charges for the Landau-Ginzburg orbifold of the torus.

For our forthcoming computation it is convenient to rewrite the superpotential (2.10) in terms of two independent coupling constants, $g_{0}$ and $g_{1}$, which in turn appear in the coupling tensor, $c_{i j k}$ :

$$
W=g_{0}\left(\sum_{i=1}^{3}\left(\Phi^{i}\right)^{3}\right)+g_{1}\left(-3 \Phi^{1} \Phi^{2} \Phi^{3}\right)=\sum_{i, j, k} c_{i j k} \Phi^{i} \Phi^{j} \Phi^{k} .
$$

The couplings, $c_{i j k}$, are symmetric in all indices and are given by

$$
c_{i j k}=\left\{\begin{aligned}
g_{0} & \text { for } i=j=k \\
-\frac{g_{1}}{2} & \text { for } i \neq j, j \neq k, i \neq k \\
0 & \text { else }
\end{aligned}\right.
$$

Note that the original complex structure coupling, $a$, is now identified with

$$
a=\frac{g_{1}}{g_{0}}
$$

The parameter, $a$, in the superpotential, $W$, is related to the standard complex structure modulus, $\tau$, of the torus via the relation 42

$$
j(\tau)=\left(\frac{3 a\left(a^{3}+8\right)}{a^{3}-1}\right)^{3}
$$


Here $j(\tau)$ denotes the modular invariant $j$-function of the torus. As in the minimal models, $\tau$ appears as the flat coordinate. Naively, the overall scaling of $g_{0}$ and $g_{1}$ are not important since only their ratio gives the complex structure modulus, $\tau$, of the torus. However, to parametrize the marginal deformations of the LandauGinzburg superpotential in terms of flat coordinates, it is necessary to adjust the normalization of the Landau-Ginzburg superpotential by an appropriate 'flattening' factor in order to ensure the vanishing of the Gauss-Manin connection [43]. For the cubic superpotential (2.11) the appropriate normalization is given by $g_{0}=q_{\mathrm{f}}^{-1}$, $g_{1}=q_{\mathrm{f}}^{-1} a$, where the 'flattening' factor is [43]

$$
q_{\mathrm{f}}(\tau)=\sqrt{\frac{1-a^{3}(\tau)}{3 a^{\prime}(\tau)}}=\frac{1}{3 \sqrt{2 \pi i}} \frac{\eta(\tau)}{\eta^{3}(3 \tau)} .
$$

We will find later that this choice leads to simplifications in the open-string sector as well.

\section{Landau-Ginzburg models with boundary}

In order to describe branes in the Landau-Ginzburg theory (2.2), we consider worldsheets, $\Sigma$, with boundaries, $\partial \Sigma$. As we will see the worldsheets relevant for our analysis have a single boundary. In other words $\Sigma$ has the topology of a disk, which we can map to the complex upper-half plane with coordinates $(x, y)$ with $x \in(-\infty,+\infty)$ and $y \in[0,+\infty)$. Thus, the single boundary, $\partial \Sigma$, is just the line $y=0$. Here we are interested in B-branes and therefore the boundary conditions on the worldsheet, $\Sigma$, must be compatible with the B-twist. From a superspace point of view this means that the two-dimensional $(2,2)$ superspace of the bulk theory reduces to the one-

dimensional boundary superspace (with two Grassmann coordinates $\theta=\frac{\theta^{-}-\theta^{+}}{\sqrt{2}}$ and $\bar{\theta}=\frac{\bar{\theta}^{-}-\bar{\theta}^{+}}{\sqrt{2}}$ ). Then a chiral superfield, $\Phi$, restricted to the boundary, $\partial \Sigma$, becomes a boundary chiral superfield, $\Phi_{\partial}$, which obeys the boundary chirality constraint

$$
\bar{D} \Phi_{\partial}=0 \quad \text { with } \quad \bar{D}=\frac{\partial}{\partial \bar{\theta}}-i \theta \partial_{x} .
$$

This constraint implies that at the boundary the boson, $\phi$, and the fermion, $\tau$, are the non-vanishing components of the restricted multiplets, $\Phi_{\partial}$, which have the expansion

$$
\Phi_{\partial}=\phi+\sqrt{2} \theta \tau-i \theta \bar{\theta} \partial_{x} \phi .
$$


Varying the Landau-Ginzburg action (2.2) with respect to the supercharge to be preserved at the boundary, one finds, however, that the bulk superpotential, $W$, generates a non-vanishing boundary term, which is often called the Warner term [44]

$$
\delta_{\epsilon} S \sim \lambda \int_{\partial \Sigma} d x \bar{\epsilon} \sum_{i} \partial_{i} W(\phi) \tau^{i}+\text { h.c. . }
$$

Here $\epsilon, \bar{\epsilon}$ are the infinitesimal fermionic parameters of the supersymmetry variation. In order to preserve the B-type supersymmetry there are two possibilities to proceed. (i) Impose boundary conditions on the bulk superfields, $\Phi$, such that the Warner term vanishes. This corresponds to choosing boundary conditions that imply $W=0$ on the boundary [44:10].

(ii) Add boundary superfields with a boundary action whose supersymmetry variation cancels the Warner term [11,12]. This approach naturally leads to the Kontsevich's description of B-branes in terms of matrix factorizations of the superpotential [4:5].

Recent work has provided evidence for the equivalence of the first possibility to a subclass of matrix factorizations [26,27]. This subclass can be represented by boundary superfields and in the two examples we consider, generate all other matrix factorizations by tachyon condensation [29,45].

\subsection{Matrix Factorizations}

We will now briefly review matrix factorizations from the viewpoint of adding boundary superfields. Given a factorization of the superpotential, $W$, of the form

$$
W(\phi)=\sum_{a} J^{a}(\phi) E_{a}(\phi), \quad a=1, \ldots, k,
$$

we construct a boundary action, which compensates the Warner term (3.3). This is achieved in terms of $k$ boundary fermionic superfields, $\Pi_{a}$, which obey the superspace constraint

$$
\bar{D} \Pi_{a}=\lambda E_{a}\left(\Phi_{\partial}\right) .
$$

The fermionic boundary multiplets, $\Pi_{a}$, are comprised of boundary fermions, $\pi_{a}$, and bosonic auxiliary fields, $\ell_{a}$. The kinetic terms for these fields are given by:

$$
S_{\mathrm{kin}}=\int_{\partial \Sigma} d x \int d^{2} \theta \sum_{a} \bar{\Pi}^{a} \Pi_{a} .
$$


In component fields the auxiliary fields, $\ell_{a}$, appear only algebraically, whereas the kinetic terms of the boundary fermions, $\pi_{a}$, give rise to the one-dimensional Dirac equation. Finally, we also add a boundary superpotential to the boundary action

$$
S_{J}=\int_{\partial \Sigma} d x \int d \theta \sum_{a} \Pi_{a} J^{a}\left(\Phi_{\partial}\right)+\text { h.c. . }
$$

Due to the modified chirality constraint (3.5) this boundary superpotential is not supersymmetric by itself. It is straight forward to check that due to the factorization (3.4) the supersymmetry variation of the boundary superpotential cancels the Warner term (3.3). Hence the boundary superpotential, $J$, together with the constraint (3.5) imposed on the superfields, $\Pi_{a}$, are the important ingredients, which are needed to preserve the B-type supersymmetry in the Landau-Ginzburg theory with boundaries.

Since the bulk action (2.2) together with the boundary action (3.6) and (3.7) does now preserve the B-type supercharge, we can perform the B-twist and obtain the topological B-model with boundaries. To the bulk BRST operator (2.6) we now need to add the boundary BRST operator, $\mathcal{Q}_{\partial}$, which acts on the boundary fields, $\pi_{a}$ and $\bar{\pi}^{a}$, as 3

$$
\mathcal{Q}_{\partial} \pi_{a}=\lambda E_{a}(\phi), \quad \mathcal{Q}_{\partial} \bar{\pi}^{a}=J^{a}(\phi)
$$

In the boundary action, $S_{\text {kin }}$, the fermionic fields, $i \bar{\pi}^{a}$, are conjugate to the fermionic fields, $\pi_{a}$. Hence, in the canonically quantized boundary theory the boundary fermions obey the canonical anti-commutation relation

$$
\left\{\pi_{a}, \bar{\pi}^{b}\right\}=\delta_{a}^{b}
$$

Therefore the boundary BRST operator, $\mathcal{Q}_{\partial}$, can be written as

$$
\mathcal{Q}_{\partial}^{\lambda}=J+\lambda E,
$$

in terms of the operators, $J$ and $E$, defined by

$$
J(\phi, \pi)=\sum_{a} J^{a}(\phi) \pi_{a}, \quad E(\phi, \bar{\pi})=\sum_{a} E_{a}(\phi) \bar{\pi}^{a} .
$$

3 Here we have eliminated the auxiliary fields, $\ell_{a}$ and $\bar{\ell}^{a}$. 
This structure of the boundary BRST operator is now directly related to the description of B-branes in terms of matrix factorizations. Namely, choosing a matrix representation for the Clifford algebra (3.8) generated by the boundary fermions, $\pi_{a}$ and $\bar{\pi}^{a}$, we obtain a $2^{k} \times 2^{k}$-matrix representation of the boundary BRST operator, $\mathcal{Q}_{\partial}$. Furthermore, in the basis, in which the chirality matrix, $\gamma$, is diagonal

$$
\gamma=\left(\begin{array}{cc}
\mathbf{1}_{2^{k-1} \times 2^{k-1}} & 0 \\
0 & -\mathbf{1}_{2^{k-1} \times 2^{k-1}}
\end{array}\right)
$$

the boundary BRST operator, $\mathcal{Q}_{\partial}$, can be expressed in terms of two $2^{k-1} \times 2^{k-1}$ matrices, $G^{\lambda}(\phi)$ and $F^{\lambda}(\phi)$ :

$$
\mathcal{Q}_{\partial}^{\lambda}=\left(\begin{array}{cc}
0 & G^{\lambda}(\phi) \\
F^{\lambda}(\phi) & 0
\end{array}\right)
$$

From the definition (3.10) of the boundary BRST operator we learn that the matrix, $\mathcal{Q}_{\partial}$, squares to the superpotential, $\lambda W$. This is equivalent to imposing

$$
F^{\lambda}(\phi) \cdot G^{\lambda}(\phi)=G^{\lambda}(\phi) \cdot F^{\lambda}(\phi)=\lambda W(\phi) \mathbf{1}_{2^{k-1} \times 2^{k-1}}
$$

which is a matrix factorization in the Kontsevich sense [4]. Hence such matrix factorizations (3.13) of the superpotential yield an equivalent description of the boundary BRST operator (3.10).

The cohomology of the boundary BRST operator (3.8) gives rise to the openstring operators in the topological theory, i.e. these operators are non-trivial cohomology elements with respect to the differential, $\mathcal{D}^{\lambda}$, which acts upon an operator, $\Psi$, as

$$
\mathcal{D}^{\lambda}(\Psi)=\left[\mathcal{Q}_{\partial}^{\lambda}, \Psi\right]_{ \pm}
$$

The commutator applies for bosonic operators whereas the anti-commutator is taken for fermionic operators.

When each of the $J^{a}$ and $E_{a}$ are quasi-homogeneous, the boundary fermionic multiplets can be assigned $R$-charges. Further, the cohomology of $\mathcal{D}^{\lambda}$ can be shown

4 At first glance it seems that there are only $2^{k-1} \times 2^{k-1}$-matrix factorizations. However, these are often equivalent to lower-dimensional matrix factorizations by condensing trivial brane anti-brane pairs 229,45]. 
to be independent of $\lambda$. However, the limit $\lambda \rightarrow 0$ can be singular and the cohomology at $\lambda=0$ need not agree with the cohomology for non-zero $\lambda$. To study this aspect, we observe that the boundary operators, $J$ and $E$, obey $[\{J, J\}, \Psi]_{ \pm}=[\{E, E\}, \Psi]_{ \pm}=$ $[\{J, E\}, \Psi]_{ \pm}=0$. Hence the individual operators, $J$ and $E$, give rise to two commuting cohomology differentials, $[J, \cdot]_{ \pm}$and $[E, \cdot]_{ \pm}$. Therefore in computing the cohomology at $\lambda=0$, that is to say the cohomology of the differential, $\mathcal{D}^{\lambda=0} \equiv[J, \cdot]_{ \pm}$, we get a first approximation for the cohomology with respect to $\mathcal{D}^{\lambda \neq 0}$. However, in correcting the cohomology elements perturbatively in $\lambda$, some cohomology elements of $\mathcal{D}^{\lambda=0}$ might drop out at a finite order in $\lambda$ because at this order they cannot be completed perturbatively to a cohomology element of the full BRST operator, $\mathcal{D}^{\lambda \neq 0}$. This process of recursively completing the cohomology elements corresponds to evaluation the spectral sequence of the double complex associated to the commuting differentials, $[J, \cdot]_{ \pm}$and $[E, \cdot]_{ \pm}$. In the two examples that we consider in this paper, it turns out that the limit $\lambda \rightarrow 0$ is not singular.

We will now discuss the boundary conditions and the corresponding matrix factorizations in the two examples of interest.

\subsection{Example 1: The A-type minimal model}

The only possible boundary condition in the Landau-Ginzburg model (2.8) that flows in the infrared to the $A$-type minimal model is the Dirichlet boundary condition [10],

$$
\Phi_{\partial}=c
$$

The constant, $c$, is equal to any root of $W_{A_{k}}$, and therefore we obtain $\left.W_{A_{k}}\right|_{\partial \Sigma} \equiv 0$. Furthermore, as discussed in Appendix A, the Dirichlet boundary condition implies that the remaining components of the chiral superfield, $\Phi$, (and its conjugate, $\bar{\Phi}$ ) form

in the absence of the superpotential a fermionic boundary chiral superfield, $\bar{\Xi}$, with the superfield expansion

$$
\bar{\Xi}=\bar{\xi}-\sqrt{2} \theta i \partial_{y} \bar{\phi}-i \theta \bar{\theta} \partial_{x} \bar{\xi}
$$


The boundary chiral superfield, $\bar{\Xi}$, gives rise to interactions at the boundary, $\partial \Sigma$, of the worldsheet, $\Sigma$. The only possible boundary interaction, which is relevant in the topological theory, is given by

$$
S_{\partial}=X \int_{\partial \Sigma} d x \int d \theta \bar{\Xi}+\text { h.c. . }
$$

We have introduced a boundary coupling constant, $X$, which we will eventually promote to a coupling matrix so as to include Chan-Paton factors.

The boundary condition (3.16) is equivalent to the rank-one matrix factorization with $J=\phi$ and $E=\phi^{k+1} /(k+2)$, which again is equivalent to the $L=0$ boundary state in the corresponding boundary CFT [5]. We observe that all three descriptions have precisely one boundary deformation with identical $R$-charges. This is a simple check on the equivalence of those three formulations. A more intuitive way to understand the connection to matrix factorizations is to see that $\bar{\xi}$ effectively plays the rôle of the boundary fermion, $\pi$, and the boundary condition (3.16) becomes the low-energy condition $J=0$. As we will discuss further in our second example, in order to match the boundary deformations in these different formulations, a certain spectral sequence associated to the double complex of the boundary operators, $J$ and $E$, needs to collapse. This is similar to the situation arising from the double complex associated to the bulk BRST operator (2.6).

It is known that the matrix factorizations for the $L>0$ boundary states in the CFT can be obtained by tachyon condensation of a suitable number of $L=0$ matrix factorizations [29]. In our approach several boundary components can be added by using Chan-Paton indices to distinguish the boundaries. The various coupling constants now become coupling matrices carrying Chan-Paton indices. For example, in addition to imposing Dirichlet boundary conditions the $L=1$ matrix factorization is represented by enhancing the coupling constant, $X$, to the $2 \times 2$ coupling matrix, $\underline{X}$. Then the off-diagonal entries of $\underline{X}$ representing 'tachyons' that mediate the formation of the $L=1$ bound state. 


\subsection{Example 2: The 'Long' branes on the cubic torus}

\begin{tabular}{|c|c|c|}
\hline Fermionic operators & $\pi_{1}, \pi_{2}, \pi_{3}$ & $\pi_{1} \pi_{2} \pi_{3}$ \\
\hline Bosonic operators & $\pi_{1} \pi_{2}, \pi_{1} \pi_{3}, \pi_{2} \pi_{3}$ & $\mathbf{1}$ \\
\hline
\end{tabular}

Table 3. Fermionic and bosonic operators in the cohomology of the differential, $\mathcal{D}^{\lambda=0}$. All these operators extend to the cohomology of $\mathcal{D}^{\lambda \neq 0}$, where the cohomology elements in the columns are related by Serre duality.

On the cubic torus we focus in this work on the brane configuration which is associated to a $4 \times 4$-matrix factorization generated by three boundary fermions, $\pi_{i}$ :

$$
\mathcal{Q}_{\partial}^{\lambda}=\phi^{i} \pi_{i}+\lambda \partial_{i} W(\phi) \bar{\pi}^{i}, \quad i=1,2,3
$$

This matrix factorization describes the 'long' branes, $L_{a}$, in the terminology of refs. [32,45]. 5 The cohomology at $\lambda=0$ is readily computed and is summarized in Table 3. Furthermore for the matrix factorization (3.19) it is easy to check that all the cohomology elements for $\lambda=0$ can be recursively completed to cohomology elements for finite values of $\lambda$, and hence the dimension of the cohomology group is not changed by the Landau-Ginzburg superpotential. This simplification need not occur for a generic matrix factorization, e.g. for the 'short' branes of refs. [32, 45] the boundary cohomology of the free theory is larger than the cohomology in the presence of the Landau-Ginzburg superpotential. Here we concentrate on the 'long' branes, $L_{a}$, where this subtlety does not play a rôle, but we come back to the general situation elsewhere.

5 One obtains three 'long' branes, $L_{a}$, since one really considers equivariant matrix factorizations, which introduces the additional $\mathbb{Z}_{3}$-valued label $a$ [46,45]. 


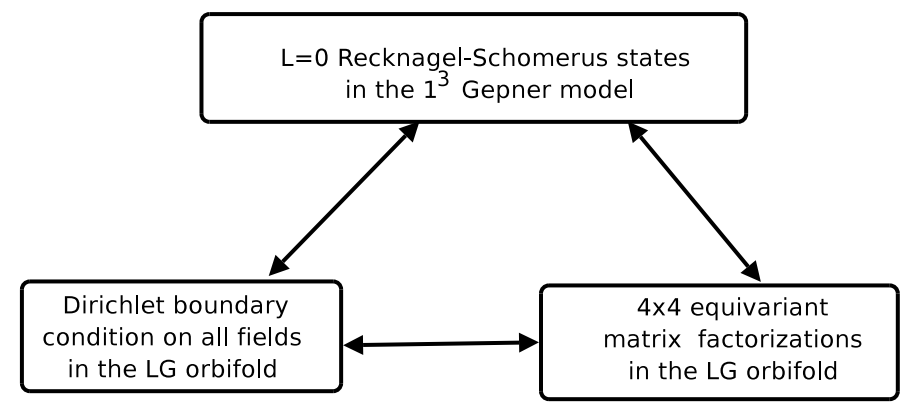

Fig. 1: The relationship between the different constructions of the 'long' branes on the torus, $\mathcal{T}$, at the Gepner point in the Kähler moduli space.

Similarly to the equivalent descriptions of branes in the $A$-type minimal model there are also different formulations for the 'long' branes, $L_{a}$, on the cubic torus as summarized in Fig. 1. Here we mainly describe the 'long' branes by imposing Dirichlet boundary conditions. Therefore, in order to close the circle we sketch the relationship of the $4 \times 4$-matrix factorization (3.19) of the torus to the brane description obtained by imposing Dirichlet boundary conditions on all three chiral bulk multiplets, $\Phi^{i}$. As discussed in the previous subsection and in Appendix A, Dirichlet boundary conditions correspond to $\phi^{i}=0$ and $\tau^{i}=0$, and on the boundary they reduce the bulk chiral multiplets, $\Phi^{i}$, to a chiral fermionic boundary multiplets, $\bar{\Xi}_{i}=\left(\bar{\xi}_{i}, \partial_{y} \bar{\phi}_{i}\right)$ [25].

With these boundary fermions, we can now construct boundary operators. In particular there are two kinds of fermionic operators, namely $\psi=\underline{X}^{i} \bar{\xi}_{i}$ and $\Omega=$ $\underline{U} \epsilon^{i j k} \bar{\xi}_{i} \bar{\xi}_{j} \bar{\xi}_{k}$, which match the results from matrix factorization summarized in Table 3 . Furthermore, from the scaling (2.3) of the Landau-Ginzburg superpotential we know that the chiral bulk superfields, $\Phi^{i}$, have $R$-charge $+\frac{2}{3}$ ( $c f$. Table 2). This allows us to infer that the operators, $\psi$ and $\Omega$, have $R$-charges $+\frac{1}{3}$ and +1 , and therefore they correspond to relevant operators and a marginal operator, respectively.

In order to describe interactions among several branes the couplings, $\underline{X}^{i}$ and $\underline{U}$, are not just taken to be scalars, but instead they are enhanced to Chan-Paton matrices. In the absence of the superpotential the Landau-Ginzburg orbifold of the torus degenerates to the $\mathbb{C}^{3} / \mathbb{Z}_{3}$ orbifold, which has three fractional $D 0$-branes [26]. 
Here these three branes are distinguished by assigning to the boundary three different Chan-Paton labels, and the matrix-valued couplings, $\underline{X}^{i}$ and $\underline{U}$, become

$$
\underline{X}^{i}=\left(\begin{array}{ccc}
0 & x_{12}^{i} & 0 \\
0 & 0 & x_{23}^{i} \\
x_{31}^{i} & 0 & 0
\end{array}\right) \quad, \quad \underline{U}=\left(\begin{array}{ccc}
u_{1} & 0 & 0 \\
0 & u_{2} & 0 \\
0 & 0 & u_{3}
\end{array}\right)
$$

The off-diagonal nature of the Chan-Paton matrices, $\underline{X}^{i}$, implies that the operators, $\psi$, are boundary condition changing operators while the diagonal nature of the matrix, $\underline{U}$, implies that the operator, $\Omega$, is a boundary condition preserving operator. The boundary operators with their Chan-Paton matrices reflect the quiver diagram associated to the three fractional $D 0$-branes of the $\mathbb{C}^{3} / \mathbb{Z}_{3}$ orbifold [47]. In the matrix factorization picture, the orbifolding of the Landau-Ginzburg model implies that we must deal with equivariant matrix factorizations and the Chan-Paton indices are the equivariant labels [46, 48, 45].

The boundary interaction that is relevant to the topological theory on the cubic torus is given by

$$
S_{\partial}=\mu \underline{X}^{i} \int_{\partial \Sigma} d x \int d \theta \bar{\Xi}_{i}+\nu \underline{U} \int_{\partial \Sigma} d x \int d \theta \epsilon^{i j k} \bar{\Xi}_{i} \bar{\Xi}_{j} \bar{\Xi}_{k}+\text { h.c. },
$$

where $\mu$ and $\nu$ are two constant parameters that we introduce for future convenience.

\section{The topological partition function and the effective superpotential}

The main focus of this work is to compute in the context of Landau-Ginzburg models the topological partition function, $Z_{\text {disk }}^{\text {top }}$, for worldsheets with the topology of a disk. This partition function is holomorphic in both the bulk and boundary couplings, and it is directly related to the worldvolume superpotential, $\mathcal{W}$, of the physical open-superstring theory. In this section we introduce the topological partition

disk function, $Z_{\text {disk }}^{\text {top }}$, and show its connection to the effective $D$-brane superpotential, $\mathcal{W}$. We conclude this section by illustrating these concepts with the $A$-type minimal model. 


\subsection{Interpreting the topological partition function}

We will now argue that the topological partition function on the disk, $Z_{\text {disk }}^{\text {top }}$, computes the effective superpotential, $\mathcal{W}$, on the worldvolume of the corresponding brane configuration. Based on the results of Shatashvili and Witten [49,50], it was conjectured in ref. [51] and proved in ref. [52] that the tree-level effective action of the open-superstring field theory is given by the superstring disk partition function. Thus, in the topological theory, which describes the holomorphic subsector of the physical theory, it is natural to make the identification of the topological partition function of the Landau-Ginzburg model, $Z_{\text {disk }}^{\text {top }}$, with the holomorphic effective $D$-brane superpotential, $\mathcal{W}$ [13]:

$$
Z_{\text {disk }}^{\text {top }}(t, u)=\mathcal{W}(t, u)
$$

Here the the complex parameters, $t$ and $u$, symbolically represent the bulk and boundary couplings, respectively.

Obstructions to $D$-brane deformations give rise to yet another relation to the topological partition function. In the context of branes given as matrix factorizations in Landau-Ginzburg models, obstructions in deforming matrix factorization can be encoded in a superpotential [21,8,22, which we denote by $\mathcal{W}_{\text {def. }}$. This means that matrix factorizations exist only on the sublocus in the space of bulk and boundary couplings, $t$ and $u$, where $d \mathcal{W}_{\text {def }}(t, u)=0$. For the two examples that we study, we will find that up to field redefinitions

$$
Z_{\text {disk }}^{\text {top }}(t, u)=\mathcal{W}_{\text {def }}(t, u)
$$

where $u$ parametrizes deformations of the boundary BRST operator, $\mathcal{Q}_{\partial}$, by fermionic operators, whereas $t$ captures the (bulk) deformations of the Landau-Ginzburg superpotential, $W$. This approach to superpotentials provides an interpretation that works also for the non-geometric examples such as the $A$-type minimal model, and it is also in agreement with the suggestion of refs. [53,54] that the lifting of moduli arising from vector bundles is encoded in effective superpotentials.

Finally, $Z_{\text {disk }}^{\text {top }}$ has a third interpretation as the generating function of (symmetrized) correlation functions [28,29, 55. This follows formally from the path-integral representation of the topological disk partition function because taking derivatives with respect to the coupling constants and then setting the couplings to zero is equivalent to computing (symmetrized) correlation functions. 


\subsection{A perturbative computation of the effective superpotential}

The main advantage of the Landau-Ginzburg model is that some of the computations can be reduced to those involving free fields. In other words, one can treat the Landau-Ginzburg superpotential, $W$, perturbatively. This means that we go to the limit $\lambda=0$, in which the Landau-Ginzburg superpotential, $W$, vanishes, and then we perform a perturbative expansion in the formal coupling constant, $\lambda$. In doing so we take advantage of the free-field formulation and in particular following ref. [25] we also use the bulk-boundary propagators of free-fields. We restrict our attention to Dirichlet boundary conditions, which are the simplest boundary conditions in Landau-Ginzburg models, and which, as discussed in the previous section, are compatible with the perturbative limit, $\lambda=0$, due to the fact that the boundary cohomology does not become singular. Thus altogether one has

$$
\mathcal{W} \equiv Z_{\text {disk }}^{\text {top }}=\sum_{n=0}^{\infty} \lambda^{n}\left\langle\left\langle\frac{\left(S_{W}\right)^{n}}{n !} P\left(e^{\mathcal{S}_{\partial}}\right)\right\rangle\right\rangle \equiv \sum_{n=0}^{\infty} \lambda^{n} \mathcal{W}_{n}
$$

where $\langle\langle\cdots\rangle\rangle$ denotes the free-field correlators on the disk, $\Sigma$, which has been mapped to the upper half plane. Further, $S_{W}=\int_{\Sigma} d^{2} x \int d^{2} \theta W(\Phi)$ and $S_{\partial}$ represent bulk and boundary interactions. The latter appear path-ordered, $P(\cdot)$, because, as explained in the previous section, including Chan-Paton factors renders the boundary interactions, $S_{\partial}$, matrix-valued.

\subsection{The A-minimal model}

We will now proceed to compute the effective $D$-brane superpotential, $\mathcal{W}$, or

equivalently the topological disk partition function, $Z_{\text {disk }}^{\text {top }}$, in the Landau-Ginzburg model for the $A$-minimal model. This is in many ways the simplest family of LandauGinzburg models. Naively, the topological partition function vanishes because of fermionic zero-modes. Therefore only correlators which saturate those zero-modes contribute to the topological partition function. In addition we also need to gaugefix the $\operatorname{PSL}(2, \mathbb{R})$ invariance of the upper-half plane. So, we compute $\frac{\partial^{2} \mathcal{W}}{\partial \lambda \partial X}$, which is according to eq. (4.3) a two-point function involving one bulk operator and one boundary operator. The $P S L(2, \mathbb{R})$ invariance is fixed by choosing these two operators as unintegrated (zero-form) operators. To be specific, we place the bulk operator at 
$\left(x_{0}, y_{0}\right)$ and the boundary operator at $x=+\infty$. The boundary zero-form operator is given by $\bar{\xi}(+\infty)$ and thus also provides for the required $\bar{\xi}$-zero mode. Thus, we will compute

$$
\frac{\partial^{2} \mathcal{W}}{\partial \lambda \partial X}=\sum_{j=0}^{k+2}\left\langle V_{j}^{(0)}\left(x_{0}, y_{0}\right) \bar{\xi}(+\infty)\right\rangle
$$

where $V_{j}^{(0)}=g_{k+2-j}(t)(\phi)^{j}$ is the bulk zero-form operator.

In computing the free-field correlation functions, non-vanishing correlators appear only if the total $R$-charge of all operators equals $\hat{c}=(1-\alpha)$. Further, with the exception of the fermionic zero modes all the fields that appear in the operators must be contracted with (some) other field in order to yield a non-zero answer. A simple consideration of these two conditions shows that for this example, correlators involving more than one bulk insertion vanish. Thus, $\mathcal{W}_{1}$ is the only non-vanishing contribution to the effective superpotential, $\mathcal{W}$, as defined in eq. (4.3), and we find

$$
\frac{\partial^{2} \mathcal{W}}{\partial \lambda \partial X}=\sum_{j=0}^{k+2} g_{k+2-j}(t) \frac{X^{j}}{j !}\left\langle\left\langle(\phi)^{j}\left(x_{0}, y_{0}\right) P\left(\frac{1}{\sqrt{2}} \prod_{k=1}^{j} \int_{-\infty}^{+\infty} d x_{k} i \partial_{y} \bar{\phi}\left(x_{k}\right)\right) \bar{\xi}(\infty)\right\rangle\right\rangle .
$$

Since we assume for now that the coupling, $X$, is a scalar, path-ordering of the boundary operators is not necessary.

The next task is to explicitly evaluate the correlator (4.5). The bulk-boundary propagator is given by

$$
\left\langle\phi\left(x_{0}, y_{0}\right) i \partial_{y} \bar{\phi}\left(x_{k}\right)\right\rangle=\mathcal{L}_{y}\left(x_{0}-x_{k}, y_{0}\right),
$$

where the Lorentzian $\mathcal{L}_{y}(x, y)$ is defined to be

$$
\mathcal{L}_{y}(x, y) \equiv \frac{y}{x^{2}+y^{2}} .
$$

There are $j$ ! contractions that are possible between the bulk operator and the $j$ boundary insertions. As each integral gives a factor of $\pi$, we are led to the following result after summing over all terms in the bulk Landau-Ginzburg superpotential

$$
\frac{\partial^{2} \mathcal{W}}{\partial \lambda \partial X}=\sum_{j=0}^{k+2} g_{k+2-j}(t)\left(\frac{\pi X}{\sqrt{2}}\right)^{j}
$$


Finally, the last expression can be integrated to

$$
\mathcal{W}=\frac{\sqrt{2} \lambda}{\pi} \sum_{j=0}^{k+2} g_{k+2-j}(t) \frac{(\pi X / \sqrt{2})^{j+1}}{j+1} .
$$

The above computation may seem to be valid only when the coupling, $X$, is a scalar and not a coupling matrix, $\underline{X}$. Since then one should treat the path-ordering carefully by also taking into account the off-diagonal entries of $\underline{X}$, which correspond to boundary condition changing operators. However, a careful treatment of the pathordering, which comes into play when the boundary coupling, $X$, is enhanced to a matrix, $\underline{X}$, shows that the sole effect is taken care of by the replacement

$$
X^{j+1} \longrightarrow \operatorname{Tr}\left(\underline{X}^{j+1}\right) \text {. }
$$

Then eq. (4.9) becomes in terms of the coupling matrix, $\underline{X}$,

$$
\mathcal{W}=\frac{\sqrt{2} \lambda}{\pi} \sum_{j=0}^{k+2} g_{k+2-j}(t) \frac{\operatorname{Tr}\left[(\pi \underline{X} / \sqrt{2})^{j+1}\right]}{j+1} .
$$

\subsection{A second alternative computation}

We will now evaluate $\frac{\partial^{3} \mathcal{W}}{\partial X^{3}}$ and verify that the result is compatible with eq. (4.11). The motivation for carrying out this computation is two-fold: First, as we will see, the analysis is not quite the same as in section 4.3. Therefore, it serves as a non-trivial check on the previous result for the effective superpotential, $\mathcal{W}$. Second, it provides for

a simple example, which allows us to illustrate the combinatorics involved in relating correlators to effective superpotential terms.

We identify $\frac{\partial^{3} \mathcal{W}}{\partial X^{3}}$ with a three-point function of three boundary operators, which, in order to fix the $\operatorname{PSL}(2, \mathbb{R})$ symmetry, are zero forms located at $x=0,1$ and $+\infty$. Thus, we expect

$$
\frac{\partial^{3} \mathcal{W}}{\partial X^{3}}=\mathbf{2}\langle\bar{\xi}(0) \bar{\xi}(1) \bar{\xi}(+\infty)\rangle
$$

The need for the factor of two in the above expression is subtle. The simplest way to understand this is to study the precise relationship between the first computation in section 4.3 , where one bulk operator and one boundary operator were chosen as zero 
forms, and the current computation, where three boundary operators are chosen as zero forms. These two different choices can be related to each other by Ward identities as was shown in refs. [56,28]. From this analysis it follows that we need to sum over two configurations, which are obtained by exchanging the operators fixed at 0 and 1. 6 Since the operators at 0 and 1 are identical, there appears a factor of two in the symmetrized correlator (4.12).

As before the only non-vanishing contribution occurs for a single bulk insertion, which is now a two-form operator and takes the form ( $c f$. Appendix A):

$$
V_{j}^{(2)}=\frac{j(j-1)}{2} g_{k+2-j}(t) \phi^{j-2} \tau \xi .
$$

Further, the total $R$-charge constraint implies that we need to have $(j-2)$ integrated boundary insertions. The relevant fermionic free-field propagators are

$$
\langle\tau(x, y) \bar{\xi}(w)\rangle=\mathcal{L}_{y}(x-w, y), \quad\langle\xi(x, y) \bar{\xi}(w)\rangle=\mathcal{L}_{x}(x-w, y),
$$

in terms of the Lorentzians

$$
\mathcal{L}_{y}(x-w, y) \equiv \frac{y}{(x-w)^{2}+y^{2}}, \quad \mathcal{L}_{x}(x-w, y) \equiv \frac{x-w}{(x-w)^{2}+y^{2}} .
$$

Then carrying out the various contractions, one obtains:

$$
\begin{aligned}
\frac{\partial^{3} \mathcal{W}}{\partial X^{3}}= & \mathbf{2} \sum_{j} \frac{j(j-1)}{2} g_{k+2-j}(t)\left(\frac{X}{\sqrt{2}}\right)^{j-2} \int_{0}^{+\infty} d y \int_{-\infty}^{+\infty} d x\left(\prod_{i=1}^{j-2} \int_{-\infty}^{+\infty} d x_{i} \mathcal{L}_{x}\left(x-x_{i}, y\right)\right) \\
& \times\left(\mathcal{L}_{y}(x-1, y) \mathcal{L}_{x}(x, y)-\mathcal{L}_{x}(x-1, y) \mathcal{L}_{y}(x, y)\right) .
\end{aligned}
$$

The two terms in the second line arise from the two possible fermionic contractions. The combinatoric factor $(j-2)$ ! originates from the number of bosonic contractions, which cancels the factor $(j-2)$ ! in the denominator. The latter factor appears from expanding the exponential in the disk partition function (4.3) or in other words from 'pulling down' $(j-2)$ boundary insertions. Finally, the $(j-2)$ boundary integrations are easy to carry out and yield the factor $\pi^{j-2}$, whereas the bulk integration contributes $\frac{\pi^{2}}{2}$. Putting all these numerical factors together, we arrive at

$$
\frac{\partial^{3} \mathcal{W}}{\partial X^{3}}=\lambda \sum_{j} j(j-1) \frac{\pi^{2}}{2} g_{k+2-j}(t)\left(\frac{\pi X}{\sqrt{2}}\right)^{j-2} .
$$

This result is clearly consistent with the effective superpotential (4.11) computed in the previous subsection.

\footnotetext{
6 The operator at $+\infty$ is identical in both situations.
} 


\subsection{Proof of the HLL conjecture}

For A-minimal models at low values of $k$ Herbst, Lerche and Lazaroiu (HLL) explicitly solve the consistency conditions on open-closed amplitudes such as the $A_{\infty^{-}}$ constraints, the bulk-boundary crossing symmetry and the Cardy constraint [28,29]. Based on this analysis, they conjectured for the $A$-minimal models a formula for the generating function of tree-level open-string amplitudes. The HLL formula precisely matches the generating function (4.11) computed in section 4.3, and hence this computation can be thought of as a proof of the HLL conjecture. In fact, the generating function (4.11) is precisely the action for the holomorphic matrix model considered in ref. [29].

Let us illustrate the connection to the HLL formula with a simple example. For the $L=1$ boundary state of the $A$-minimal model at level $k=3$ the explicit computation of HLL yields

$$
\mathcal{W}_{H L L}\left(u_{1}, u_{2}, t\right)=\sum_{j=0}^{5} g_{5-j}(t) h_{j+1}\left(u_{1}, u_{2}\right),
$$

where the functions, $g_{l}(t)$ (with $g_{1}=0$ ), depending on the flat bulk coordinates, $t$, are defined in eq. (2.8). The functions, $h_{j}(u)$, are specific homogeneous functions of degree $j$, while the boundary variables, $u_{1}$ and $u_{2}$, have assigned degrees 1 and 2 , respectively. For example, the first few functions, $h_{j}$, are

$$
h_{0}=1, \quad h_{1}=u_{1}, \quad h_{2}=u_{2}+\frac{u_{1}^{2}}{2}, \quad h_{3}=u_{1} u_{2}+\frac{u_{1}^{3}}{3}, \quad \ldots .
$$

In order to compare the generating function (4.11) to eq. (4.18) we evaluate the generating function (4.11) at level $k=3$ and choose for the coupling matrix, $\underline{X}$, a $2 \times 2$ matrix so as to model the $L=1$ bound state. Then we find a precise agreement

with the superpotential $\mathcal{W}_{H L L}$ if we set $h_{j}=\frac{\pi^{j} \operatorname{Tr}\left(\underline{X}^{j}\right)}{2^{j / 2} j}$ and if we further identify the two invariants of the coupling matrix, $\underline{X}$, with the variables, $u_{1}$ and $u_{2}$ :

$$
u_{1} \equiv \frac{\pi \operatorname{Tr}(\underline{X})}{\sqrt{2}}, \quad u_{2} \equiv-\frac{\pi^{2} \operatorname{det}(\underline{X})}{2} .
$$




\section{The perturbative computation for the cubic torus}

In this section we evaluate the topological disk partition function for the 'long' branes on the cubic torus. As before we carry out the computation by using the perturbative techniques applied in the previous section. However, compared to the $A$-type minimal model the analysis on the torus is more involved since the whole disk partition function turns out to be a series of an infinite number of different graphs. This feature is due to the fact that the 'long' branes, $L_{a}$, possess an open-string modulus generated by a marginal open-string operator. We evaluate only the first view terms in this series explicitly, but in addition we are able to extract certain properties of the series to all orders.

\subsection{The effective superpotential of the cubic torus}

In order to obtain the effective superpotential, $\mathcal{W}$, of the torus we first compute the correlation function, $\frac{\partial^{2} \mathcal{W}}{\partial \lambda \partial \nu}$, which reads

$$
\frac{\partial^{2} \mathcal{W}}{\partial \lambda \partial \nu}=\left\langle V_{W}^{(0)}\left(x_{0}, y_{0}\right) 3 ! U \overline{\boldsymbol{\xi}}_{\mathbf{1}} \bar{\xi}_{\mathbf{2}} \bar{\xi}_{\mathbf{3}}(+\infty)\right\rangle
$$

Recall that the parameters, $\lambda$ and $\nu$, are the couplings arising in the bulk and boundary interactions (2.2) and (3.21), and differentiating with respect to these parameters pulls down one bulk and one boundary insertions. They are taken as the bulk zero form, $V_{W}^{(0)}=W(\phi)$, at $\left(x_{0}, y_{0}\right)$ and the boundary zero form at $+\infty$ in order to fix the $P S L(2, \mathbb{R})$ symmetry of the upper half plane. This particular correlation function turns out to be a convenient choice to saturate the three fermionic zero modes, $\bar{\xi}_{i}$, $i=1,2,3$, which we have indicated in the correlators in bold face letters.

Next, in the perturbative computation, we need to expand the exponential (4.3) containing the bulk and boundary interactions $S_{W}$ and $S_{\partial}$, where we keep only terms that satisfy the total $R$-charge condition. A simple analysis shows that the only nonvanishing terms occur for three integrated $\psi^{(1)}$ insertions and an equal but arbitrary number of integrated $V_{W}^{(2)}$ and $\Omega^{(1)}$ insertions. Thus, unlike in the $A$-minimal model, we find that the effective superpotential, $\mathcal{W}$, as defined in eq. (4.3) receives contributions from all terms, $\mathcal{W}_{n}$. This is due to the fact that both the boundary operator, 
$\Omega$, and the bulk operator, $W(\phi)$, are marginal, which means that their integrated one-form and two-form versions do not change the $R$-charge of the correlator.

To summarize, the contribution to $\mathcal{W}_{n}$ arises from $n$ bulk insertion, $n \Omega$ insertions and three $\Psi$ insertions. So the perturbative computation, written in terms of freefield correlators, leads to the following infinite sum after taking into account the total $R$-charge condition:

$$
\begin{aligned}
\frac{\partial^{2} \mathcal{W}}{\partial \lambda \partial \nu}= & \sum_{n=1}^{\infty} \frac{(\lambda \nu)^{n-1} \mu^{3}}{[(n-1) !]^{2}} \times \\
& \left\langle\left\langle V_{W}^{(0)}\left(\int_{\Sigma} V_{W}^{(2)}\right)^{n-1} P\left[\left(\int_{\partial \Sigma} \psi^{(1)}\right)^{3}\left(\int_{\partial \Sigma} \Omega^{(1)}\right)^{n-1}\right] \underline{U} \bar{\xi}_{1} \bar{\xi}_{2} \bar{\xi}_{3}(+\infty)\right\rangle\right\rangle .
\end{aligned}
$$

In the above expression, all boundary integrals run over the full $x$-axis as follows from the Dyson formula for a path-ordered exponential. As we demonstrate in Appendix C the final result depends only on the combination $\left(u_{1}+u_{2}+u_{3}\right)$, which is the trace of the Chan-Paton matrix, $\underline{U}$. So for computational simplicity, we can set $u_{1}=u_{2}=u_{3}=\frac{u}{3}$ such that the matrix, $\underline{U}$, becomes proportional to the identity matrix, $\mathbf{1}_{3 \times 3}$. Therefore the path ordering, involving the boundary operators, $\Omega$, becomes easier to handle. Putting in the explicit form of the various operators, we obtain

$$
\begin{aligned}
\frac{\partial^{2} \mathcal{W}}{\partial \lambda \partial \nu} & =\frac{u}{3} \sum_{m=0}^{\infty} \frac{(3 ! \lambda \nu u)^{m} \mu^{3}}{[m !]^{2}}\left(\frac{1}{\sqrt{2}}\right)^{3 m+3} \times \\
& \left\langle\left\langle\left[c \phi^{3}\right]\left(\int_{\Sigma}[c \phi \tau \xi]\right)^{n-1} P\left[\left(\int_{\partial \Sigma}\left[\underline{X} \partial_{y} \bar{\phi}\right]\right)^{3}\left(\int_{\partial \Sigma}\left[\epsilon \bar{\xi} \bar{\xi} \partial_{y} \bar{\phi}\right]\right)^{n-1}\right] \overline{\boldsymbol{\xi}}_{1} \bar{\xi}_{2} \bar{\xi}_{3}(+\infty)\right\rangle\right\rangle,
\end{aligned}
$$

where we have used the short-hand notation $\left[c \phi^{3}\right]$ for $c_{i j k} \phi^{i} \phi^{j} \phi^{k}$ and so on. The next step in the computation is to carry out the various bulk-boundary contractions and to evaluate the integrals

$$
\frac{\partial^{2} \mathcal{W}}{\partial \lambda \partial \nu}=\frac{u}{3} \sum_{m=0}^{\infty} \frac{(3 ! \lambda \nu u)^{m} \mu^{3}}{[m !]^{2}}\left(\frac{\pi}{\sqrt{2}}\right)^{3 m+3}\left(\frac{1}{2}\right)^{m} \sum_{r} \mathfrak{c}_{m+1, r} \mathcal{C}_{m+1, r}
$$

where every boundary integral gives a factor of $\pi$ and the bulk integral gives a factor of $\frac{\pi^{2}}{2}$ ( $c f$. Appendix C). The sum, $\sum_{r}$, runs over all distinct contractions, which can be given a graphical representation. The numerical coefficient, $\mathfrak{c}_{n, r}$, captures the the 
combinatorial multiplicity of the graph $r$ at order $n$ in the parameter $u$. That is to say the coefficients, $\mathfrak{c}_{n, r}$, count the number of ways a given graph can be obtained. Finally, the factor, $\mathcal{C}_{n, r}$, describes the group-theoretic contribution of each graph, which arises from summing over the couplings, $c_{i j k}$ and $\epsilon^{i j k}$, of the marginal (bulk and boundary) vertex operators and from tracing over the couplings, $\underline{X}^{i}$, of the relevant boundary operators. The technical details concerning these issues are collected in Appendix B.

Finally, we integrate eq. (5.4) to obtain the expansion in the effective superpotential, $\mathcal{W}$

$$
\mathcal{W}=\mathcal{W}_{0}+\frac{1}{3 \gamma} \sum_{n=1}^{\infty} \frac{\gamma^{n} u^{n}}{(n !)^{2}} \sum_{r} \mathfrak{c}_{n, r} \mathcal{C}_{n, r}
$$

where $\gamma \equiv 3(\pi / \sqrt{2})^{3}$ and where we have set $\lambda=\mu=\nu=1$. Obviously, the analyzed correlation function (5.1) does not capture the term, $\mathcal{W}_{0}$, as it appears in eq. (5.5) as an integration constant. Therefore we need to compute $\mathcal{W}_{0}$ separately, which we turn to in the next subsection.

\subsection{Computing the zero-order term, $\mathcal{W}_{0}$}

The correlation function (5.1) considered in the previous subsection is insensitive to the term $\mathcal{W}_{0}$ of the expansion (4.3). Hence, in order to compute $\mathcal{W}_{0}$, we consider

now the correlation function, $\frac{\partial^{3} \mathcal{W}}{\partial \mu^{3}}$, which is equal to the three-point function of three boundary operators, $\psi^{(0)}$. As discussed in the context of the $A$-type minimal model in section 4.4, the precise identification involves a factor of two:

$$
\frac{\partial^{3} \mathcal{W}}{\partial \mu^{3}}=\mathbf{2}\left\langle\psi^{(0)}(0) \psi^{(0)}(1) \psi^{(0)}(+\infty)\right\rangle \text {. }
$$

Since, we are only interested in the term, $\mathcal{W}_{0}$, i.e. the term with no bulk and no $\Omega$ insertions, we evaluate the correlation function (5.6) at $u=0$ and obtain:

$$
\begin{aligned}
\left.\frac{\partial^{3} \mathcal{W}_{0}}{\partial \mu^{3}}\right|_{u=0} & =\mathbf{2}\left\langle\left\langle\psi^{(0)}(0) \psi^{(0)}(1) \psi^{(0)}(+\infty)\right\rangle\right\rangle \\
& =2 \operatorname{Tr}\left(\underline{X}^{i} \underline{X}^{j} \underline{X}^{k}\right) \epsilon_{i j k}\left\langle\left\langle\bar{\xi}_{1}(0) \bar{\xi}_{2}(1) \bar{\xi}_{3}(+\infty)\right\rangle\right\rangle \\
& =2 \operatorname{Tr}\left(\underline{X}^{i} \underline{X}^{j} \underline{X}^{k}\right) \epsilon_{i j k} .
\end{aligned}
$$


Here the three $\bar{\xi}$ zero-modes are provided by the operator insertions at 0,1 and $+\infty$. Integrating the last expression and setting $\mu=1$ we arrive at:

$$
\mathcal{W}_{0}=\frac{1}{3} \operatorname{Tr}\left(\underline{X}^{i} \underline{X}^{j} \underline{X}^{k}\right) \epsilon_{i j k}
$$

In fact, one can also compute $\mathcal{W}_{1}$ starting from the correlation function (5.6) and verify that it agrees with the result obtained in eq. (5.5). However, we are not able to carry out the integrals that appear in computing $\mathcal{W}_{n}$ for $n>2$ from the correlation function (5.6). Hence it is not possible to compare the higher order terms.

\subsection{Gross features of the effective D-brane superpotential, $\mathcal{W}$}

Several features of the structure of the effective $D$-brane superpotential, $\mathcal{W}$, may already be extracted without getting into computational details. First of all, as previously discussed one can obtain non-vanishing correlators only for the overall correct $R$-charge and only for an equal number of bulk and $\Omega$ insertions. This observation establishes the structure of the terms, $\mathcal{W}_{n}$, in the expansion (4.3) of $\mathcal{W}$ :

$$
\mathcal{W}_{n} \propto\left(g_{0} u\right)^{n} \operatorname{Tr}\left(\underline{X}^{i} \underline{X}^{j} \underline{X}^{k}\right) f_{i j k}
$$

Here $f_{i j k}$ denotes a $S U(3)$ tensor. It is constructed from the $n^{\text {th }}$ symmetric power of the fully symmetric third-rank $S U(3)$ tensor, $c_{i j k}$, which enters in eq. (5.9) through the $n$ bulk insertions. Further, the cyclic property of the trace implies that $\operatorname{Tr}\left(\underline{X}^{i} \underline{X}^{j} \underline{X}^{k}\right)$ must either be proportional to $\epsilon^{i j k}$, which transforms as a $S U(3)$ singlet, or must be again a symmetric third-rank tensor of $S U(3)$. Thus this simple group-theoretic analysis tells us that $\mathcal{W}_{n}$ can only be non-zero if the symmetric tensor product, $S^{n}(\square \square)$, contains either a singlet or a symmetric third-rank tensor, $\square \square$.

By decomposing the trace, $\operatorname{Tr}\left(\underline{X}^{i} \underline{X}^{j} \underline{X}^{k}\right)$, into its $S U(3)$ representations and by comparing with the representations appearing in $S^{n}(\square \square)$ we can even determine which parts of the trace can possibly appear in the term, $\mathcal{W}_{n}$. Expanding

\footnotetext{
7 We abbreviate the symmetric third-rank tensor of $S U(3)$ by its Young tableau,
} 
$\operatorname{Tr}\left(\underline{X}^{i} \underline{X}^{j} \underline{X}^{k}\right)$, it is useful to reorganize it into three kinds of terms, which we will call $\kappa_{111}, \kappa_{123}$ and $\kappa_{132}$, and which are defined as

$$
\begin{aligned}
& \kappa_{111}(\underline{X})=\frac{1}{3} \sum_{i} \operatorname{Tr}\left(\underline{X}^{i} \underline{X}^{i} \underline{X}^{i}\right)=x_{12}^{1} x_{23}^{1} x_{31}^{1}+x_{12}^{2} x_{23}^{2} x_{31}^{2}+x_{12}^{3} x_{23}^{3} x_{31}^{3}, \\
& \kappa_{123}(\underline{X})=\operatorname{Tr}\left(\underline{X}^{1} \underline{X}^{2} \underline{X}^{3}\right)=x_{12}^{1} x_{23}^{2} x_{31}^{3}+x_{12}^{3} x_{23}^{1} x_{31}^{2}+x_{12}^{2} x_{23}^{1} x_{31}^{3}, \\
& \kappa_{132}(\underline{X})=\operatorname{Tr}\left(\underline{X}^{1} \underline{X}^{3} \underline{X}^{2}\right)=x_{12}^{1} x_{23}^{3} x_{31}^{2}+x_{12}^{2} x_{23}^{1} x_{31}^{3}+x_{12}^{3} x_{23}^{1} x_{31}^{2} .
\end{aligned}
$$

Then the combination $\left(\kappa_{123}-\kappa_{132}\right)$ forms the $S U(3)$ singlet, while $\kappa_{111}$ and $\left(\kappa_{123}+\right.$ $\left.\kappa_{132}\right)$ are components of the $S U(3)$ representation $\square \square$. Due to the special structure of the bulk couplings, $c_{i j k}$, given in eq. (2.12), only these two components appear in the effective superpotential, $\mathcal{W}$.

First, we observe that the reducible representation, $S^{2}(\square \square)$, contains neither a singlet nor the representation $\square$, and therefore we conclude $\mathcal{W}_{2}=0$. Further, using the computer algebra package $\mathrm{LiE}$ [57], we have checked up to $n=20$, that singlets appear only in the decomposition of $S^{2 n}(\square \square)$ while the representation arise only in $S^{2 n+1}(\square \square)$. In the next section we present an argument that shows that this pattern is indeed true to all orders $n$. Note also that the first instance when the multiplicities in $S^{k}(\square \square)$ of the two representations in question is greater than one occurs for the first time at $k=7$.

Finally, up to an overall proportionality constant, we can write the first six terms in $\mathcal{W}$ by explicitly working out the $f_{i j k}$, and we find:

$$
\begin{aligned}
& \mathcal{W}_{0}=\mathcal{I}_{0}\left(-3\left(\kappa_{123}-\kappa_{132}\right)\right) \\
& \mathcal{W}_{1}=\mathcal{I}_{1}\left(3 \kappa_{111}-\frac{3}{2} a\left(\kappa_{123}+\kappa_{132}\right)\right) g_{0} u, \\
& \mathcal{W}_{2}=0 \\
& \mathcal{W}_{3}=\mathcal{I}_{3}\left(-\frac{9}{2} a^{2} \kappa_{111}+\left(3-\frac{3}{4} a^{3}\right)\left(\kappa_{123}+\kappa_{132}\right)\right)\left(g_{0} u\right)^{3}, \\
& \mathcal{W}_{4}=\mathcal{I}_{4}\left(-\frac{9}{2} a^{4}-36 a\right)\left(\kappa_{123}-\kappa_{132}\right)\left(g_{0} u\right)^{4}, \\
& \mathcal{W}_{5}=\mathcal{I}_{5}\left(\left(\frac{3}{8} a^{4}+3 a\right) \kappa_{111}-\left(\frac{3}{16} a^{5}+\frac{3}{2} a^{2}\right)\left(\kappa_{123}+\kappa_{132}\right)\right)\left(g_{0} u\right)^{5}, \\
& \mathcal{W}_{6}=\mathcal{I}_{6}\left(\frac{9}{4} a^{6}-45 a^{3}-18\right)\left(\kappa_{123}-\kappa_{132}\right)\left(g_{0} u\right)^{6},
\end{aligned}
$$


with

$$
\mathcal{I}_{0}=\frac{1}{3}, \quad \mathcal{I}_{1}=\frac{2 \gamma}{3}, \quad \mathcal{I}_{3}=-\frac{4 \gamma^{3}}{9}, \quad \mathcal{I}_{4}=-\frac{2 \gamma^{4}}{9}
$$

where $\gamma \equiv 3(\pi / \sqrt{2})^{3}$. In Appendix B, we discuss the details of this computation. But we want to emphasis here that the group-theoretical structure, i.e. the appearance of the appropriate traces (5.10) and the vanishing of the term, $\mathcal{W}_{2}$, arises also directly by explicitly evaluating the correlation function (5.1) as outlined in section 5.1 and in Appendix B.

\section{Modular properties of the toroidal effective superpotential}

In section 5 we perturbatively computed the effective superpotential, $\mathcal{W}$, for the 'long' branes, $L_{a}$, of the Landau-Ginzburg model with the cubic superpotential (2.11). In this analysis, however, we have not really used the geometry of the underlying torus, $\mathcal{T}$. Thus, in this section we exploit the modular properties of the torus, $\mathcal{T}$, in order to extract further properties of the effective superpotential, $\mathcal{W}$.

It is well-known that the Landau-Ginzburg theory (2.11) corresponds in the large radius limit of the Kähler moduli space to a supersymmetric $\sigma$-model, for which the target space is the torus, $\mathcal{T}$, given as the hypersurface, $W=0$, in the projective space, $\mathbb{P}^{2}$ [37. The parameter, $a$, in the superpotential, $W$, parametrizes the complex structure of this hypersurface, and it is related to the standard complex structure modulus, $\tau$, of the torus via the relation (2.14). Note that for a torus with complex structure, $\tau$, there are generically twelve different possible values for the parameter, $a$, which are the roots of the order twelve polynomial associated to eq. (2.14). All these distinct roots describe in the large radius limit identical $\sigma$-models, and hence the associated Landau-Ginzburg theories are also equivalent.

In our setup, where we treat the Landau-Ginzburg superpotential (2.11) perturbatively, different ratios (2.13) of the couplings $g_{0}$ and $g_{1}$, yet ratios associated via eq. (2.14) to the same modulus, $\tau$, should also give rise to equivalent correlation functions. $\mathrm{B}$ In particular, as the boundary operator, $\Omega$, is independent of the structure of the perturbative superpotential (2.11), the superpotential contributions, $\mathcal{W}_{n}$, which

8 At least as long as the couplings $g_{0}$ and $g_{1}$ are small. 
are graded by the number of $\Omega$ insertions, must also be related order by order for equivalent Landau-Ginzburg theories.

In order to study the properties of the superpotential terms, $\mathcal{W}_{n}$, we rephrase the question in an appropriate language. First of all, the relationship (2.14) defines the parameter, $a$, to be a modular function of $\Gamma[3]$, i.e. the function, $a$, is invariant under the action of the group $\Gamma[3]$. Moreover, the different roots of the polynomial associated to eq. (2.14) are permuted under the Galois group of the polynomial. The Galois group of this polynomial is the tetrahedral group $\mathcal{T}_{12} \simeq P S L\left(2, \mathbb{Z}_{3}\right) \simeq P S L(2, \mathbb{Z}) / \Gamma[3]$ of index 12. From this perspective using the properties of the modular function, $a$, and by requiring invariance of the correlators, $\mathcal{W}_{n}$, we want to determine the modular transformation behavior of the Chan-Paton traces, $\kappa_{111},\left(\kappa_{123}+\kappa_{132}\right),\left(\kappa_{123}-\kappa_{132}\right)$, and of the coupling product, $g_{0} u$.

We study the group, $\mathcal{T}_{12}$, by looking at its generators, $S$ and $T$, which act upon the complex structure, $\tau$, as 9

$$
S: \tau \rightarrow-\frac{1}{\tau}, \quad T: \tau \rightarrow \tau+1
$$

Then eq. (2.14) encodes the transformation behavior of the modular function, $a$, to be 42

$$
S: a \rightarrow \frac{a+2}{a-1}, \quad T: a \rightarrow \rho^{2} a,
$$

where $\rho=e^{\frac{2 \pi i}{3}}$. It is straight forward to check that for a given root, $a$, these two transformations generate all the other roots of the polynomial associated to eq. (2.14).

The next task is to deduce the modular properties of the remaining quantities. From the superpotential term, $\mathcal{W}_{0}$, in eq. (5.11) we readily see that the Chan-Paton trace, $\left(\kappa_{123}-\kappa_{132}\right)$, is invariant under the group, $\mathcal{T}_{12}$, i.e.

$$
S: \kappa_{123}-\kappa_{132} \rightarrow \kappa_{123}-\kappa_{132}, \quad T: \kappa_{123}-\kappa_{132} \rightarrow \kappa_{123}-\kappa_{132}
$$

This allows us directly to deduce from the term, $\mathcal{W}_{4}$, in eq. (5.11) the group action on the product, $g_{0} u$ :

$$
S: g_{0} u \rightarrow-i \frac{(a-1)}{\sqrt{3}} g_{0} u, \quad T: g_{0} u \rightarrow \rho^{-2} g_{0} u
$$

9 Since the group $P S L(2, \mathbb{Z})$ is generated by the group elements $S$ and $T$, also the tetrahedral group, $\mathcal{T}_{12}$, as a quotient group of $\operatorname{PSL}(2, \mathbb{Z})$, is generated by $S$ and $T$. 
Finally, we determine from the superpotential contribution, $\mathcal{W}_{1}$, the modular properties of the Chan-Paton traces, $\kappa_{111}$ and $\left(\kappa_{123}+\kappa_{132}\right)$,

$$
\begin{aligned}
& S:\left(\begin{array}{c}
\kappa_{111} \\
\kappa_{123}+\kappa_{132}
\end{array}\right) \rightarrow\left(\begin{array}{c}
\frac{i}{\sqrt{3}}\left(\kappa_{111}+\left(\kappa_{123}+\kappa_{132}\right)\right) \\
\frac{i}{\sqrt{3}}\left(2 \kappa_{111}-\left(\kappa_{123}+\kappa_{132}\right)\right)
\end{array}\right), \\
& T:\left(\begin{array}{c}
\kappa_{111} \\
\kappa_{123}+\kappa_{132}
\end{array}\right) \rightarrow\left(\begin{array}{c}
\rho^{2} \kappa_{111} \\
\kappa_{123}+\kappa_{132}
\end{array}\right) .
\end{aligned}
$$

Note that in this analysis we have only used the terms $\mathcal{W}_{0}, \mathcal{W}_{1}$ and $\mathcal{W}_{4}$ in eq. (5.11) in order to arrive at the transformation rules (6.3), (6.4) and (6.5). The other terms in the list (5.11) serve as non-trivial checks and confirm the stated results.

On the other hand we can also use the derived modular properties so as to constrain the general structure of the superpotential terms, $\mathcal{W}_{n}$. In particular we now show that the Chan-Paton trace, $\left(\kappa_{123}-\kappa_{132}\right)$, does only appear in $\mathcal{W}_{n}$ for even values of $n$. As discussed in the previous section a contribution to $\mathcal{W}_{n}$ involving $\left(\kappa_{123}-\kappa_{132}\right)$ has the general structure

$$
\left(\sum_{k=0}^{n} \alpha_{k}(a-1)^{k}\right)\left(g_{0} u\right)^{n}\left(\kappa_{123}-\kappa_{132}\right),
$$

with numerical coefficients, $\alpha_{k}$. Invariance of this expression with respect to the generator, $S$, constrains the coefficients, $\alpha_{k}$ :

$$
0=\sum_{k=0}^{n} \frac{\alpha_{k}}{(\sqrt{3})^{n-k}}\left((\sqrt{3})^{n-k}(a-1)^{k}+i^{n}(\sqrt{3})^{k}(a-1)^{n-k}\right) .
$$

Note that in this formula $i^{n}$ becomes \pm 1 for even integers, $n$, which implies that the polynomials of the coefficients, $\alpha_{k}$ and $\alpha_{n-k}$, in eq. (6.7) are linearly dependent. Therefore the condition (6.7) can always be non-trivially fulfilled for even values of $n$. However, for odd integers, $n$, we get $i^{n}= \pm i$, and as a consequence the polynomials of all the coefficients, $\alpha_{k}$, in eq. (6.7) are linearly independent, which enforces all the coefficients, $\alpha_{k}$, to be zero. Thus the Chan-Paton trace, $\left(\kappa_{123}-\kappa_{132}\right)$, can never contribute to odd orders in the effective superpotential, $\mathcal{W}$. By similar arguments one also shows that the other Chan-Paton traces, $\kappa_{111}$ and $\left(\kappa_{123}+\kappa_{132}\right)$, do not appear 
at even orders in the effective superpotential, $\mathcal{W} .10$ Hence this analysis confirms to all orders in $n$ the group-theoretical claims made in section 5.3 and we conclude that the effective superpotential, $\mathcal{W}$, possess the $\mathbb{Z}_{2}$ symmetry

$$
\left(u, \kappa_{111}(\underline{X}), \kappa_{123}(\underline{X}), \kappa_{132}(\underline{X})\right) \rightarrow\left(-u,-\kappa_{111}(\underline{X}),-\kappa_{132}(\underline{X}),-\kappa_{123}(\underline{X})\right) .
$$

In order to gain further insight into the meaning of the open-string couplings, $u$, we want to reinterpret the modular behavior of the product, $g_{0} u$. As we have discussed in section 2.2 to describe the deformations of Landau-Ginzburg superpotential, $W$, in terms of flat coordinates, we need to identify the coupling constant, $g_{0}$, with the inverse 'flattening' factor, $q_{\mathrm{f}}^{-1}$, which we introduced in eq. (2.15). From the transformation behavior of the modular functions, $a$, in eq. (6.2), we immediately deduce for the 'flattening' factor (2.15) (and, hence, also for the inverse coupling, $g_{0}^{-1}$ )

$$
S: q_{\mathrm{f}} \rightarrow \frac{\sqrt{3} i}{\tau(a-1)} q_{\mathrm{f}}, \quad T: q_{\mathrm{f}} \rightarrow \rho^{2} q_{\mathrm{f}} .
$$

Comparing with eq. (6.4) we identify the coupling, $g_{0}$, with the inverse factor, $q_{\mathrm{f}}^{-1}$, and then we deduce for the open-string parameter, $u$, the modularity:

$$
S: u \rightarrow \frac{u}{\tau}, \quad T: u \rightarrow u .
$$

Note that these transformations of the open-string coupling, $u$, match the modular properties of a point on the torus, $\mathcal{T}$.

Let us pause to stress the significance of this result. The perturbative treatment of the Landau-Ginzburg superpotential contains naturally the 'flattening' factor, $q_{\mathrm{f}}$, which in ref. [43] is determined by differential equations arising from the periods of the torus. Moreover, due to eq. (6.10) the open-string coupling, $u$, can be thought of as a point on the torus. Thus this parameter is the (combined) open-string modulus of the three 'long' branes, $L_{a}$, and it coincides with the flat open-string coordinate, $u$, used in refs. 32, 155. 11

10 If we take into account the transformation behavior with respect to the generator, $T$, we find that the term, $\mathcal{W}_{2}$, must also vanish by modularity.

11 In principle by the presented arguments the coupling, $u$, could still differ from the flat open-string coordinate by a multiplicative factor given by a modular invariant function. However, in the sequel we will show that the coupling, $u$, is indeed the flat coordinate. 
Furthermore, as the parameter, $u$, transforms as point on the torus and as the Chan-Paton trace, $\left(\kappa_{123}-\kappa_{132}\right)$, is according to eq. (6.3) modular invariant, the $\tau$-dependent part in the even superpotential terms, $\mathcal{W}_{2 k}$, transform as modular functions, $\tilde{G}_{2 k}$, of weight $2 k, 12$ i.e.

$$
\mathcal{W}_{2 k}=\tilde{G}_{2 k}(\tau)\left(\kappa_{123}-\kappa_{132}\right) u^{2 k},
$$

with

$$
\tilde{G}_{2 k}\left(\frac{a \tau+b}{c \tau+d}\right)=(c \tau+d)^{2 k} \tilde{G}_{2 k}(\tau) .
$$

Before concluding this section we rewrite the effective superpotential, $\mathcal{W}$, so as to explicitly show its general structure and its dependence on the bulk and boundary moduli, $\tau$ and $u$ :

$$
\mathcal{W}(\tau, u, \underline{X})=\Delta_{111}(\tau, u) \kappa_{111}(\underline{X})+\Delta_{123}(\tau, u) \kappa_{123}(\underline{X})+\Delta_{132}(\tau, u) \kappa_{132}(\underline{X}) .
$$

Note that due to the symmetry property (6.8) of the effective superpotential, $\mathcal{W}$, the functions, $\Delta_{i j k}$, obey

$$
\Delta_{111}(\tau, u)=-\Delta_{111}(\tau,-u), \quad \Delta_{123}(\tau, u)=-\Delta_{132}(\tau,-u) .
$$

\section{Mirror map and disk instantons for the 'long' A-branes on the torus}

Up to now we have performed all our computations in the topological B-model. In this section we compute the $D$-brane effective superpotential of the configuration, which is mirror symmetric to the 'long' branes of the cubic torus, $\mathcal{T}$. By comparing the effective superpotential of the A-model with the one of the B-model we are able deduce the open-string mirror map. Since the two effective superpotentials are comprised of a collection of correlation functions, the existence of a unique mirror map is also an indirect but non-trivial check on the perturbative B-model computations performed in the previous sections.

12 Due to their modular properties the functions, $\tilde{G}_{2 k}$, are proportional to the Eisenstein functions, $G_{2 k}$, for $2 \leq k \leq 5$. However, this need not be true for $k>5$. Since the Eisenstein functions, $G_{4}$ and $G_{6}$, generate all the higher weight Eisenstein functions, for instance only a particular linear combination of $G_{4}^{3}$ and $G_{6}^{2}$ coincides with the Eisenstein function, $G_{12}$, which might not be proportional to $\tilde{G}_{12}$. Finally, it is interesting to note that the multiplicity of the singlets in the symmetric tensor product, $S^{2 k}(\square \square)$, as discussed in section 5.3, coincides (at least for $2 \leq k \leq 10$ ) with the number of linearly independent modular functions of weight $2 k$. 


\subsection{The effective superpotential in the A-model}

All contributions to the effective $D$-brane superpotential, $\hat{\mathcal{W}}, 13$ on the A-model side arise from non-perturbative effects, namely from worldsheet disk instantons [58]. In general summing up the contributions of those disk instantons in a Calabi-Yau manifold is a highly non-trivial problem. However, since the mirror manifold of the torus, $\mathcal{T}$, is yet again a torus, $\hat{\mathcal{T}}$, the special Lagrangian submanifolds, which represent $A$-type $D$-branes in the geometric regime of the A-model, are just given by real lines on the torus $\hat{\mathcal{T}}$. Hence analyzing disk instantons for this toroidal geometry simply amounts to computing areas of triangles, which has been carried out in refs. [30, 31, 32, 33] 14 In order to set the stage for the next section we briefly review this analysis for the mirror branes, $\hat{L}_{a}$, of the 'long' branes, $L_{a}$.

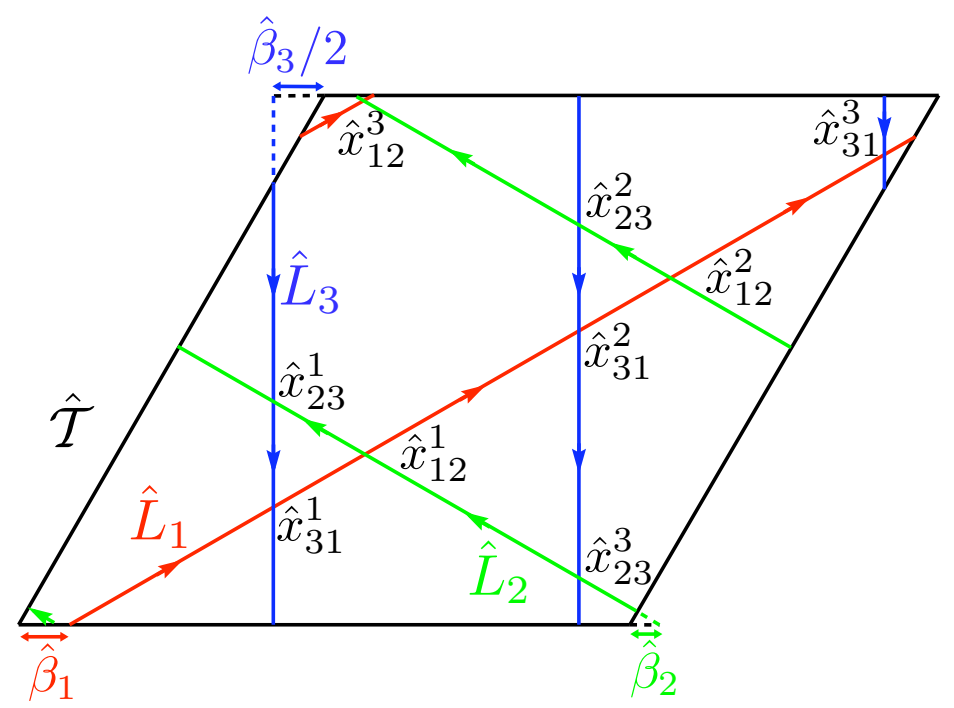

Fig. 2: The torus, $\hat{\mathcal{T}}$, is shown together with the three A-type D1branes $\hat{L}_{a}$. Their real moduli, $\hat{\beta}_{a}$, parametrize the offsets of the branes, $\hat{L}_{a}$, in the horizontal direction. The boundary changing operators, $\hat{x}_{a b}^{i}$, are located at the intersection of the branes, $\hat{L}_{a}$ and $\hat{L}_{b}$.

13 We distinguish the A-model from B-model quantities by adding a hat ^ for A-model quantities.

14 For superpotential terms involving more than three boundary changing operators one needs to compute areas of polygons [33]. 
On the B-model side we have performed our computations at the LandauGinzburg point, which is located at the point $\rho=e^{\frac{2}{3} i \pi}$ in the Kähler moduli space. As mirror symmetry exchanges the Kähler and the complex structure moduli, $(\tau, \rho) \leftrightarrow(\hat{\rho}, \hat{\tau})$, of mirror pairs, we consider the mirror torus, $\hat{\mathcal{T}}$, at $\hat{\tau}=e^{\frac{2}{3} i \pi}$ in the complex structure moduli space. Moreover, we need to identify the A-model mirror images, $\hat{L}_{a}$, of the B-model 'long' branes, $L_{a}$. At the Landau-Ginzburg point the three 'long' branes, $L_{a}$, carry the RR-charges [32,45]

$$
L_{a}: \quad\left(r, c_{1}\right)^{\mathrm{LG}}=\{(-1,1),(-1,-2),(2,1)\},
$$

where the charge vector $\left(r, c_{1}\right)$ denotes the $D 2$-brane and $D 0$-brane charge respectively. On the A-model side these charges become the winding numbers of the corresponding one-dimensional Lagrangian submanifolds of the mirror torus, $\hat{\mathcal{T}}$ [30]. This allows us to identify the A-model branes, $\hat{L}_{a}$, depicted in Fig. 2. Furthermore the boundary changing operators, $\hat{x}_{a b}^{i}$, are located at the intersections of the lines associated to the branes, $\hat{L}_{a}$ and $\hat{L}_{b}$. .

The A-model correlators are given by the sum of disk instantons bounded by the associated Lagrangian submanifolds and weighted by their (complexified) areas. These areas depend on the (real) positions, $\hat{\beta}_{a}$, of the bounding branes, $\hat{L}_{a}$, and therefore the three possible correlation functions take schematically the form [30]:

$$
C_{i j k}^{A}\left(\hat{\rho}, \hat{\alpha}_{a}, \hat{\beta}_{a}\right)=\sum_{k} e^{2 \pi i \hat{\rho} A_{i j k}^{(k)}\left(\hat{\beta}_{a}\right)} e^{2 \pi i W_{i j k}^{(k)}\left(\hat{\alpha}_{a}\right)}
$$

Here the coefficients, $A_{i j k}^{(k)}$, denote the (dimensionless) areas of the different disk instantons labeled by the index $k$, whereas the phases, $W_{i j k}^{(k)}$, are Wilson line contributions. The latter are obtained by integrating the flat connection along the circumference of the disk instanton. The connection of the brane, $\hat{L}_{a}$, is parametrized by the single real modulus, $\hat{\alpha}_{a}$, because the topology of the associated one-dimensional submanifold is a circle. Finally, note that only the correlators $C_{111}^{A}, C_{123}^{A}, C_{132}^{A}$ (and their cyclic permutations) are non-vanishing.

15 The orientation of the intersection specifies whether the corresponding boundary changing operator is fermionic or bosonic. In the following we concentrate only on fermionic boundary changing operators as their couplings appear in the effective superpotential, $\hat{\mathcal{W}}$. 


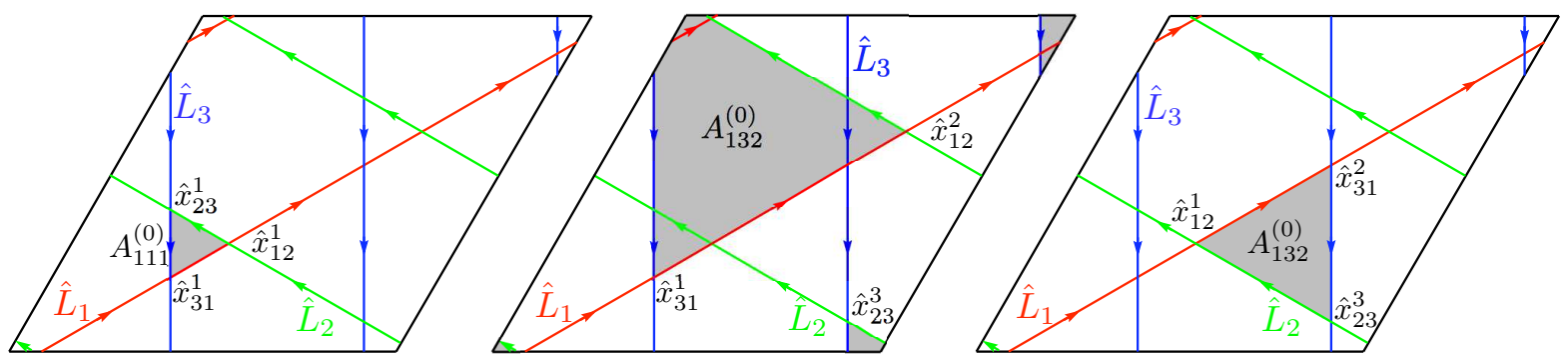

Fig. 3: For the D1-branes, $\hat{L}_{1}, \hat{L}_{2}$ and $\hat{L}_{3}$, the non-trivial correlators $\left\langle\hat{x}_{31}^{1} \hat{x}_{12}^{1} \hat{x}_{23}^{1}\right\rangle,\left\langle\hat{x}_{31}^{1} \hat{x}_{12}^{2} \hat{x}_{23}^{3}\right\rangle$ and $\left\langle\hat{x}_{12}^{1} \hat{x}_{23}^{3} \hat{x}_{31}^{2}\right\rangle$ arise from disk instantons. The figures show the disk instantons $k=0$ for these three correlation functions respectively.

The areas, $A_{i j k}^{(k)}$, of the disk instantons can be deduced by evaluating the areas of all triangles formed by the corresponding boundary changing operators in the correlators, $C_{i j k}^{A}$. The disk instantons labeled by $k=0$ are shown for the non-vanishing correlators in Fig. 3. A view steps of elementary geometry reveal for a general instanton labeled by $k \in \mathbb{Z}$

$$
A_{111}^{(k)}=\frac{3}{2}\left(k+\frac{\hat{\beta}}{3}\right)^{2}, \quad A_{123}^{(k)}=\frac{3}{2}\left(k+\frac{1}{3}+\frac{\hat{\beta}}{3}\right)^{2}, \quad A_{132}^{(k)}=\frac{3}{2}\left(k-\frac{1}{3}+\frac{\hat{\beta}}{3}\right)^{2}
$$

and for its Wilson line contributions

$$
W_{111}^{(k)}=\left(k+\frac{\hat{\beta}}{3}\right) \hat{\alpha}, \quad W_{123}^{(k)}=\left(k+\frac{1}{3}+\frac{\hat{\beta}}{3}\right) \hat{\alpha}, \quad W_{132}^{(k)}=\left(k-\frac{1}{3}+\frac{\hat{\beta}}{3}\right) \hat{\alpha} .
$$

Here we have used the definitions $\hat{\alpha}=\hat{\alpha}_{1}+\hat{\alpha}_{2}+\hat{\alpha}_{3}$ and $\hat{\beta}=\hat{\beta}_{1}+\hat{\beta}_{2}+\hat{\beta}_{3}$. Inserting eqs. (7.3) and (7.4) into the correlator (7.2) we finally arrive in terms of the complex variables $\hat{\rho}$ and $\hat{u}=\hat{\alpha}+\hat{\rho} \hat{\beta}$ at

$$
C_{i j k}^{A}(\hat{\rho}, \overline{\hat{\rho}}, u, \overline{\hat{u}})=\left(e^{\frac{2}{3} \pi i \hat{\alpha} \hat{\beta}} \hat{q}^{\frac{\hat{\beta}^{2}}{6}}\right) \cdot \Delta_{i j k}^{A}(\hat{\rho}, u)
$$

with

$$
\begin{aligned}
& \hat{\Delta}_{111}(\hat{\rho}, \hat{u})=\sum_{k \in \mathbb{Z}} \hat{q}^{\frac{3}{2} k^{2}} e^{2 \pi i k \hat{u}} \\
& \hat{\Delta}_{123}(\hat{\rho}, \hat{u})=\sum_{k \in \mathbb{Z}} \hat{q}^{\frac{3}{2}\left(k+\frac{1}{3}\right)^{2}} e^{2 \pi i\left(k+\frac{1}{3}\right) \hat{u}}, \\
& \hat{\Delta}_{132}(\hat{\rho}, \hat{u})=\sum_{k \in \mathbb{Z}} \hat{q}^{\frac{3}{2}\left(k-\frac{1}{3}\right)^{2}} e^{2 \pi i\left(k-\frac{1}{3}\right) \hat{u}},
\end{aligned}
$$


and the non-holomorphic prefactor

$$
\wp=e^{\frac{2}{3} \pi i \hat{\alpha} \hat{\beta}} \hat{q}^{\frac{\hat{\beta}^{2}}{6}}
$$

The non-holomorphic prefactor, $\wp$, will be discussed in subsection 7.3. Here, we only observe that this factor cannot appear in the holomorphic superpotential and hence the A-model superpotential, $\hat{\mathcal{W}}$, is only given in terms of the holomorphic parts $(\overline{7.6})$ :

$$
\hat{\mathcal{W}}(\hat{\rho}, \hat{u}, \underline{\hat{X}})=\hat{\Delta}_{111}(\hat{\rho}, \hat{u}) \kappa_{111}(\underline{\hat{X}})+\hat{\Delta}_{123}(\hat{\rho}, \hat{u}) \kappa_{123}(\underline{\hat{X}})+\hat{\Delta}_{132}(\hat{\rho}, \hat{u}) \kappa_{132}(\underline{\hat{X}})
$$

Here the matrices, $\underline{\hat{X}}$, denote the A-model analogs of the B-model coupling matrices, $\underline{X}$, defined in eq. (3.20).

\subsection{The mirror map and flat coordinates}

The aim of this subsection is to determine the mirror map for the 'long' branes, $L_{a}$, on the torus, $\mathcal{T}$, and the mirror D1-branes, $\hat{L}_{a}$, on the mirror torus, $\hat{\mathcal{T}}$. That is to say we construct the map between the sets of A-model and B-model variables, or, equivalently, we determine the flat coordinates on the B-model side, which are canonically identified with variables of the A-model.

In the B-model the variables, which arise from the 'long' branes, $L_{a}$, on the torus, $\mathcal{T}$, are the bulk complex structure modulus, $\tau$, the collective $D$-brane modulus, $u$, and the coupling matrices, $\underline{X}$, of the boundary changing operators, $x_{a b}^{i}$. As discussed in the previous section, the natural variables for the mirror A-model are the bulk Kähler modulus, $\hat{\rho}$, of the torus, $\hat{\mathcal{T}}$, and the complexified position modulus, $\hat{u}$, and the boundary changing matrices, $\underline{\hat{X}}$, of $D 1$-branes, $\hat{L}_{a}$.

In the closed-string sector the complex structure modulus, $\tau$, is the flat coordinate of the B-model bulk theory 43], and hence mirror symmetry identifies the A-model Kähler modulus, $\hat{\rho}$, with the B-model complex structure modulus, $\tau$. In the following for ease of notation we will often replace the A-model Kähler parameter, $\hat{\rho}$, by its mirror variable, $\tau$.

In the open-string sector we still must determine the mirror map, for which we make the following ansatz,

$$
\hat{u}=\mathcal{N}_{u}(\tau) u+u_{0}(\tau), \quad \underline{\hat{X}}=\mathcal{N}_{X}(\tau, u) \underline{X} .
$$


This ansatz is justified by two observations. First of all the open-string mirror map should not contain any dimensionful couplings. Therefore, the functional dependence between the variables, $\hat{u}$ and $u$, can only involve (on the B-model side) the moduli, $u$ and $\tau$, but not the dimensionful matrices, $\underline{X}$. Furthermore, the additive property of both the A-model variable, $\hat{u}$, and the B-model variable, $u$, implies that $\hat{u}$ and $u$ are related linearly to one another. Similarly, by dimensional arguments the coupling matrices, $\underline{\hat{X}}$ and $\underline{X}$, can only be proportional to one another with the moduli-dependent proportionality constant, $\mathcal{N}_{X}(\tau, u)$.

Our first task is to determine the the shift $u_{0}(\tau)$. This is achieved by adjusting the parameter $u_{0}$ such that the symmetry properties (6.14) of the functions $\Delta_{i j k}(u)$ coincide with those of $\hat{\Delta}_{i j k}\left(\hat{u}-u_{0}\right)$. That is to say for the functions, $\mu_{\ell}$, defined by

$\mu_{1}(\tau, \hat{u})=\hat{\Delta}_{111}\left(\tau, \hat{u}-u_{0}\right), \quad \mu_{2}(\tau, \hat{u})=\hat{\Delta}_{123}\left(\tau, \hat{u}-u_{0}\right), \quad \mu_{3}(\tau, \hat{u})=\hat{\Delta}_{132}\left(\tau, \hat{u}-u_{0}\right)$,

we require analogously to eq. (6.14) to obey the symmetries

$$
\mu_{1}(\tau, \hat{u})=-\mu_{1}(\tau,-\hat{u}), \quad \mu_{2}(\tau, \hat{u})=-\mu_{3}(\tau,-\hat{u})
$$

It is easy to check that these two conditions are simultaneously fulfilled for

$$
u_{0}(\tau)=-\frac{1}{2} \tau-\frac{1}{2} .
$$

Note that the the shift, $u_{0}(\tau)$, required to match the A-model variables with the B-model variables, has already been observed in ref. [32].

Equating the A-model superpotential, $\hat{\mathcal{W}}$, with the B-model superpotential, $\mathcal{W}$, yields with with the ansatz (7.9) for the mirror map:

$$
\mathcal{N}_{X}^{3}(\tau, u) \hat{\mathcal{W}}\left(\tau, \mathcal{N}_{u}(\tau) u+u_{0}, \underline{X}\right)=\mathcal{W}(\tau, u, \underline{X}) .
$$

This relation allows us now to determine the open-string mirror map functions, $\mathcal{N}_{u}(\tau)$ and $\mathcal{N}_{X}(\tau, u)$, explicitly. The technical details of this computation are relegated to Appendix D, where we derive for the functions, $\mathcal{N}_{u}(\tau)$ and $\mathcal{N}_{X}(\tau, u)$, the expressions

$$
\mathcal{N}_{u}=\frac{3 \mathcal{I}_{1}}{\sqrt{2 \pi i} \mathcal{I}_{0}}=\frac{9 \sqrt{-i \pi^{5}}}{2}
$$


and

$$
\mathcal{N}_{X}(\tau, u)=\sqrt[3]{\frac{i}{\eta(\tau)}} e^{-\frac{\mathcal{N}_{u}^{2}}{18} G_{2}(\tau) u^{2}+O\left(u^{4}\right)} .
$$

The higher order terms in $u$ are determined by the precise numerical coefficients of the superpotential terms, $\mathcal{W}_{2 k}$, in eq. (6.11). Here $G_{2}(\tau)$ is the second function in the Eisenstein series and it is also given by

$$
G_{2}(\tau)=-4 \pi i \frac{\eta^{\prime}(\tau)}{\eta(\tau)} .
$$

Note that determining the open-string mirror maps, $\mathcal{N}_{u}$ and $\mathcal{N}_{X}$, form eq. (7.13) is a highly over-determined problem ( $c f$. Appendix D). Hence the existence of the solution (7.14) and (7.15) is a non-trivial check on the method of computing the effective superpotential, $\mathcal{W}$, perturbatively.

\subsection{Holomorphic anomaly}

In the physical theory the non-holomorphic prefactor arises from the Kähler potential and hence is a D-term contribution [31]. Here we want to interpret this prefactor from the topological A- and B-model point of view.

In the bulk theory of the B-model the correlators receive non-holomorphic contributions due to the holomorphic anomaly, which arises at higher genus amplitudes [13]. Therefore in the B-model with boundaries we similarly expect the appearance of nonholomorphic terms. Since we have computed tree-level disk diagrams, the obtained correlators are holomorphic in the moduli. However, due to quantum $A_{\infty}$ relations the disk diagrams are ultimately linked to open one-loop cylinder amplitudes [59,33, which potentially suffer from a holomorphic anomaly. But to clarify the precise rôle of non-holomorphic terms in the quantum $A_{\infty}$ relations is beyond the scope of this work.

Due to the properties of the amplitudes on the torus we can trace back the holomorphic anomaly of the cylinder diagrams to our computation as follows. We have seen in the previous section that the disk amplitudes have well-defined modular properties, and they can be expressed in terms of the Eisenstein series, $G_{2 k}$. Therefore the associated cylinder amplitudes should also appear in terms of the functions, $G_{2 k}$. 
In practice in computing the cylinder diagrams the bosonic zero modes must be regulated. If the chosen regulator preserves the modularity in favor of holomorphicity, the second Eisenstein function $G_{2}$ is replaced by the non-holomorphic modular function $\hat{G}_{2}$. $G_{2}$ is not a modular function of weight 2 as it suffers from a modular anomaly 60. The function $\hat{G}_{2}$ is defined by:

$$
\hat{G}_{2}(\tau)=G_{2}(\tau)-\frac{\pi}{\operatorname{Im} \tau} .
$$

If we pragmatically map the holomorphic anomaly of $\hat{G}_{2}$ arising in the cylinder diagrams to the open-string mirror map, $\mathcal{N}_{X}$, which solely contains the Eisenstein function, $G_{2}$, we obtain the modified mirror map, $\tilde{\mathcal{N}}_{X}$,

$$
\mathcal{N}_{X} \rightarrow \tilde{\mathcal{N}}_{X}=q^{-\frac{1}{6} \frac{\left(\mathcal{N}_{u} u\right)^{2}}{(2 i \operatorname{Im} \tau)^{2}}} \bar{q}^{-\frac{1}{6} \frac{\left(\mathcal{N}_{u} u\right)^{2}}{(2 i \operatorname{Im} \tau)^{2}}} \mathcal{N}_{X} .
$$

Inserting now the modified mirror map into the mirror relation (7.13) the A-model superpotential becomes:

$$
\hat{\mathcal{W}}=\mathcal{N}_{X}^{-3} \mathcal{W}\left(\tau, \mathcal{N}_{u}^{-1}\left(\hat{u}-u_{0}\right)\right) \rightarrow \tilde{\wp} \hat{\mathcal{W}}=\tilde{\mathcal{N}}_{X}^{-3} \mathcal{W}\left(\tau, \mathcal{N}_{u}^{-1}\left(\hat{u}-u_{0}\right)\right)
$$

with

$$
\tilde{\wp}=q^{\frac{1}{6} \frac{\left(\hat{u}-u_{0}\right)^{2}}{(2 i \operatorname{Im} \tau)^{2}}} \bar{q}^{\frac{1}{6}} \frac{\left(\hat{u}-u_{0}\right)^{2}}{(2 i \operatorname{Im} \tau)^{2}} .
$$

Now we want to compare this prefactor with the non-holomorphic factor (7.7) on the A-model side.

In the A-model the bulk theory of the torus, $\hat{\mathcal{T}}$, depends on a choice of a base point $(\hat{\rho}, \overline{\hat{\rho}})$, where $\hat{\rho}$ and $\overline{\hat{\rho}}$ should be thought of independent variables. Moreover, the topological A-model, which localizes on worldsheet instantons, corresponds to the choice $\overline{\hat{\rho}} \rightarrow+\infty[13,61$. On the other hand the B-model of the bulk theory of the torus, $\mathcal{T}$, does also depend on a choice of base point $(\tau, \bar{\tau})$. The B-model, which is mapped by mirror symmetry to the A-model at $\overline{\hat{\rho}} \rightarrow+\infty$, is naturally identified with the B-model at $\bar{\tau} \rightarrow+\infty$ 61.

For the topological A- and B-model with boundaries we expect also a dependence on the choice of base point in the moduli space. Therefore, in addition to the base point in the bulk sector we must also specify a base point $(\hat{u}, \bar{u})$ or $(u, \hat{u})$ in the boundary sector.

The prefactor, $\wp$, of eq. (7.7) written in terms of $\hat{q}=e^{2 \pi i \hat{\rho}}$ and $\overline{\hat{q}}=e^{-2 \pi i \hat{\hat{\rho}}}$ reads:

$$
\wp=\hat{q}^{\frac{1}{6} \frac{\hat{u}^{2}}{(2 i \operatorname{Im} \tau)^{2}}} \hat{q}^{-\frac{1}{6} \frac{\bar{u}^{2}}{(2 i \operatorname{Im} \tau)^{2}}} \overline{\hat{q}}^{-\frac{1}{3} \frac{|\hat{u}|^{2}}{(2 i \mathrm{~m} \tau)^{2}}} \overline{\hat{q}}^{\frac{1}{3} \frac{\hat{u}^{2}}{(2 i \mathrm{Im} \tau)^{2}}} .
$$

This agrees with (7.20) in terms of the base points $(\hat{u}, \overline{\hat{u}}) \rightarrow\left(\hat{u}-u_{0}, 0\right)$ and $\overline{\hat{\rho}} \rightarrow \infty$ and $\overline{\hat{\tau}} \rightarrow \infty$. 


\section{Conclusions}

In this paper we have computed the topological partition function in LandauGinzburg B-models on the disk by treating the worldsheet superpotential perturbatively. We have argued that the topological disk partition function computes effective $D$-brane superpotentials. In two examples we have illustrated that the effective superpotentials obtained by this method are compatible with known results in the literature. Furthermore, our approach is not limited to the two considered examples, but instead is also applicable in physically more interesting theories such as Landau-Ginzburg models for Calabi-Yau threefolds.

The novel feature of the torus example was the appearance of a marginal boundary operator. In comparing with the topological mirror A-model, the open-string mirror map became a function of both closed and open-string moduli, $\tau$ and $u$. An open question is whether this open-string mirror map satisfies a partial differential equation in $\tau$ and $u$, which can be derived in the B-model from first principles without making reference to the mirror A-model. In a similar vein, it would be interesting to find a derivation for the heat equation, which is satisfied by the theta functions that make up the effective $D$-brane superpotential in the example of the torus.

We have also worked out the relationship between simple boundary conditions and matrix factorizations. Matrix factorizations have a huge gauge invariance which can complicate the analysis as the number of variables increases. By directly imposing these simple boundary conditions, as pursued in this paper, the gauge redundancies of matrix factorizations do not appear. This might be a useful starting point in deriving Ward identities which potentially could lead to the type of partial differential equations mentioned earlier.

In the two considered examples we have imposed Dirichlet boundary conditions on all fields in the Landau-Ginzburg model. It is, however, of interest to extend the presented computation to situations where Neumann boundary conditions do also arise for some linear combinations of the Landau-Ginzburg fields. This occurs, for instance, for the 'short' branes in the Landau-Ginzburg model of the cubic torus. We hope to pursue this issue in the future. 


\section{Acknowledgments}

We would like to thank Ilka Brunner, Stefan Fredenhagen, Matthias Gaberdiel, K.S. Narain and Henning Samtleben for helpful comments and useful correspondences. We are especially thankful to Wolfgang Lerche and Nick Warner for many enlightening discussions at various stages of this work. SG would also like to thank the Theory Group at CERN and the ITP at ETH for their hospitality during the course of this work.

\section{Appendix A. Bulk and boundary supermultiplets}

In our conventions the component expansion of the two-dimensional $(2,2)$ chiral superfield, $\Phi$, as defined in eq. (2.1), reads

$$
\Phi=\phi+\sqrt{2} \theta^{\alpha} \psi_{\alpha}+\theta^{\alpha} \theta_{\alpha} F
$$

Here $\phi$ is the complex bosonic field, $\psi_{+}, \psi_{-}$, are the fermionic components and $F$ is the complex auxiliary field of this chiral multiplet. Let $\epsilon=\frac{\epsilon_{+}+\epsilon_{-}}{\sqrt{2}}$ parametrize the unbroken supersymmetry of B-type boundary conditions. Then one has the following supersymmetry transformations for the components of the superfields, $\Phi^{i}$ :

$$
\begin{aligned}
\delta \phi^{i} & =-\sqrt{2} \epsilon \tau^{i} \\
\delta \tau^{i} & =i \sqrt{2} \bar{\epsilon} \partial_{x} \phi^{i} \\
\delta \xi^{i} & =i \sqrt{2} \bar{\epsilon} \partial_{y} \phi^{i}+\sqrt{2} \epsilon F^{i}, \\
\delta F^{i} & =i \sqrt{2} \bar{\epsilon}\left(\partial_{y} \tau^{i}-\partial_{x} \xi^{i}\right) .
\end{aligned}
$$

In the above equations, we have introduced the combinations $\tau^{i} \equiv\left(\psi_{+}^{i}-\psi_{-}^{i}\right) / \sqrt{2}$ and $\xi^{i} \equiv\left(\psi_{-}^{i}+\psi_{+}^{i}\right) / \sqrt{2}$ as they are more appropriate for the Landau-Ginzburg model with boundaries. These transformations imply that the $(2,2)$ chiral multiplets, $\Phi^{i}$, decompose into two multiplets, $\left(\Phi_{\partial}^{i}, \Xi^{i}\right)$, under the supersymmetry preserved by the boundary. $\Phi_{\partial}^{i}$ is a boundary chiral field while $\Xi^{i}$ is a multiplet subject to the constraint $D \Xi^{i}=\sqrt{2} F^{i}$. In the absence of a superpotential, we can set $F^{i}=0$ and then, $\Xi$ is a fermionic (boundary) chiral multiplet. 
In the topological model, the generator of the supersymmetry parametrized by $\bar{\epsilon}$ is conventionally taken to be the BRST operator, $\mathcal{Q}$. The zero-form topological observables are thus $\mathcal{Q}$-closed. In the bulk, any holomorphic function of $\phi$ is an observable. The one-form and two-form versions of the bulk operators are given by

$$
\begin{aligned}
V_{f}^{(0)} & =f(\phi), \\
V_{\partial_{x} f}^{(1)} & =\frac{-i}{\sqrt{2}}\left(\partial_{j} f\right) \tau^{j}, \\
V_{\partial_{y} f}^{(1)} & =\frac{-i}{\sqrt{2}}\left(\partial_{j} f\right) \xi^{j}, \\
V_{f}^{(2)} & =-\frac{1}{2}\left(\partial_{i} \partial_{j} f\right) \tau^{i} \xi^{j} .
\end{aligned}
$$

When we impose Dirichlet boundary conditions on all the fields in the LandauGinzburg model, the (fermionic) boundary operators for the $A$-type minimal model are given by

$$
\psi^{(0)}=X \bar{\xi}, \quad \psi^{(1)}=\frac{X}{\sqrt{2}} \partial_{y} \bar{\phi},
$$

and for the cubic torus $\left(\epsilon^{i j k}\right.$ is the totally antisymmetric tensor of $\left.S U(3)\right)$

$$
\begin{aligned}
\psi^{(0)} & =\underline{X}^{i} \bar{\xi}_{i}, & \psi^{(1)} & =\frac{1}{\sqrt{2}} \underline{X}^{i} \partial_{y} \bar{\phi}_{i}, \\
\Omega^{(0)} & =\underline{U} \epsilon^{i j k} \bar{\xi}_{i} \bar{\xi}_{j} \bar{\xi}_{k}, & \Omega^{(1)} & =\frac{3}{\sqrt{2}} \underline{U} \epsilon^{i j k} \bar{\xi}_{i} \bar{\xi}_{j} \partial_{y} \bar{\phi}_{k} .
\end{aligned}
$$

\section{Appendix B. Combinatorics for the perturbative computation of the torus}

In the computation of diagrams for the torus the combinatorics becomes easier if we choose to set one bulk insertion and one $\Omega$ insertion as a zero-form located on the boundary at $+\infty$. This insertion then provides the three $\bar{\xi}$ zero-modes as well. Thus, we have fixed the $\operatorname{PSL}(2, R)$ invariance and taken care of the fermion zeromodes. This choice can be carried out for all $\mathcal{W}_{n}$ with $n>0$. We will now consider all possible contractions for this choice when $n=1,3,4$.

In the figures, we will follow the following conventions. The filled dot indicates a bulk insertion, the unfilled dot represents an $\Omega$ insertion, the cross is a $\psi$ insertion and an open circle with a dot represents the three fermion zero-modes. Bosonic 
propagators are represented by a brown line while fermionic ones are represented by a blue line. We will indicate by $\mathcal{C}_{n}$, the group-theory factor that the graph represents and $\mathfrak{c}_{n}$, the combinatoric factor associated with the graph, i.e. the number of ways that the given graph can be obtained.
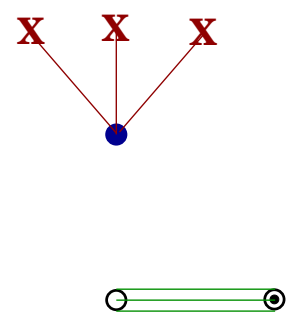

Fig. 4: $\mathcal{C}_{1}=3 \kappa_{111}-\frac{3}{2} a\left(\kappa_{123}+\kappa_{132}\right)$

The combinatoric factor associated with Fig. 4 is $\mathfrak{c}_{1}=3$ !, which is the number of ways we can carry out the $\phi-X$ contractions.

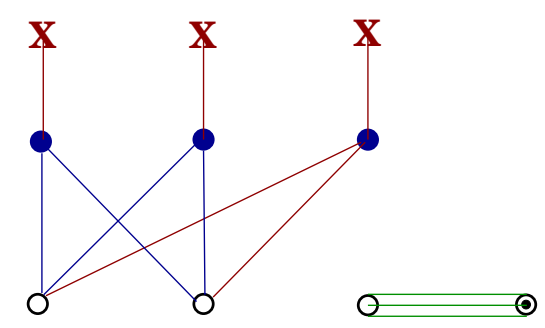

Fig. 5: $\mathcal{C}_{3}=-\frac{9}{2} a^{2} \kappa_{111}+\left(3-\frac{3}{2} a^{3}\right)\left(\kappa_{123}+\kappa_{132}\right)$

The combinatoric factor associated with Fig. 5 is $\mathfrak{c}_{3}=(3 !)(3 !) 2^{2}$. Here the first 3 ! is the number of ways we can carry out the $\phi-X$ contractions and the second 3 ! is the number of ways the three $\phi$ 's that enter the zero-form bulk insertion contract. Finally, $2^{2}$ is the number of possible fermion contractions. We assume that (we antisymmetrize by hand) $\xi \tau$ fermions that enters each bulk two-form. So the factor of 2 arises from the fermion contractions with each $\Omega$ insertion. In general, this will contribute a factor of $2^{n-1}$ to all graphs.

We find that when $n=4$, there are two possible graphs which we label $4 a$ and 4b. In $4 a$, the zero-form bulk insertion contracts with one of the $\psi$-insertions while 
in the $4 b$ it doesn't. Both graphs are identical except for this difference which shows up as distinct colorations (coming from the bosonic/fermionic propagators). So the combinatoric factors are different.

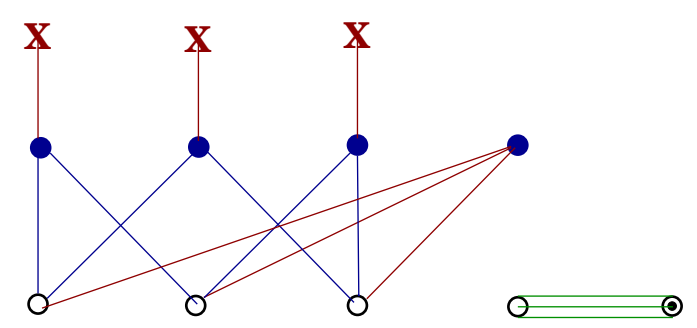

Fig. 6: $\mathcal{C}_{4 a}=-\left(\frac{3}{4} a^{4}+6 a\right)\left(\kappa_{123}-\kappa_{132}\right)$

The associated combinatoric factor is $\mathfrak{c}_{4 a}=(3 !)^{3} 2^{3}$. Again one 3 ! arises from the $\phi-X$ contractions, the second from the bulk zero-form and the last one from the fermion contractions. As explained earlier, we have $2^{4-1}$ coming from each $\Omega$ one-form.

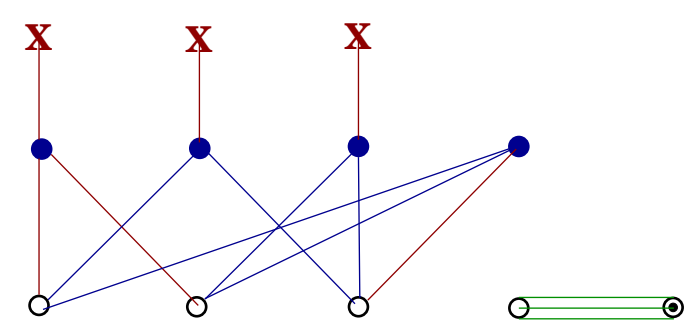

Fig. 7: $\mathcal{C}_{4 b}=-\left(\frac{3}{4} a^{4}+6 a\right)\left(\kappa_{123}-\kappa_{132}\right)$

The associated combinatoric factor is $\mathfrak{c}_{4 b}=(3 !)^{2}(3) 3 ! 2^{3}$. Here one 3 ! arises from the $\phi-X$ contractions, the second from the bulk zero-form. The 3 comes from the number of ways, one of the $\phi$ 's from the bulk-zero form can contract with one of the three $\Omega$ and the last 3 ! from the fermion contractions. So the contribution is $\left(\mathfrak{c}_{4 a} \mathcal{C}_{4 a}+\mathfrak{c}_{4 b} \mathcal{C}_{4 b}\right)$.

From $n=5$ onwards the structure is more intricate since the number of inequivalent graphs increases while their individual complexity is growing. 


\section{Appendix C. Integration of the torus correlators}

The computation of the various free-field correlators requires us to evaluate integrals involving Lorentzians $\mathcal{L}_{x}$ and $\mathcal{L}_{y}$ defined in eq. (4.15). If all the integrals were over the real line or real half line, then we could use the residue theorem to evaluate the integrals because the functions, $\mathcal{L}_{x}$ and $\mathcal{L}_{y}$, are obtained as the real and imaginary parts of a meromorphic function. However, the required path-ordering implies that this is not the case for the boundary integrals that we compute.16 So we find that we cannot typically carry out all integrations. Naively extending the limits of all integrals is not compatible with the correct addition of contributions from different path-orderings that enter the partition function and gives a vanishing answer.

There is however, one situation, where we can indeed do the integrals. As we will see, in a special limit, the integrands simplify to a collection of delta functions. This happens when we choose one bulk operator to be a zero form located at $\left(x_{0}, y_{0}\right)$ and one boundary operator to be a zero form located at $x=+\infty$ whereas all other operators are integrated ones. $P S L(2, \mathbb{R})$ invariance implies that the answer must be independent of $x_{0}$ and $y_{0}$. In particular, one can take the limit $y_{0} \rightarrow 0$. This is the limit when the unintegrated bulk insertion is taken close to the boundary of the upper half-plane. Then one finds

$$
\lim _{y_{0} \rightarrow 0} \mathcal{L}_{y}\left(w-x_{0}, y_{0}\right)=\pi \delta\left(w-x_{0}\right)
$$

It is not hard to see that in the limit, $y_{0} \rightarrow 0$, all bulk-boundary contractions involving the bulk zero-form operator reduce to such delta functions. Moreover, the resulting product of delta functions implies that any boundary operator contracted with the bulk zero form gets only a contribution if it is located at $x_{0}$ on the real line. Since delta functions are easy to integrate this technique provides a simpler way to compute, for instance, the term, $\mathcal{W}_{1}$. One may view the bulk zero-form insertion at $y_{0} \neq 0$ as a regulator replacing delta functions by Lorentzians with width $y_{0}$.

16 The path-ordering is non-trivial since the Chan-Paton matrices $\underline{X}^{i}$ and $\underline{U}$ do not commute for arbitrary $u_{i}$. They commute for $u_{1}=u_{2}=u_{3}$. 


\section{C.1. The integral for $\mathcal{W}_{3}$}

Rather than consider the most general integral, we next consider the integral that arises in computing the superpotential term, $\mathcal{W}_{3}$. Let the bulk zero form be located at $\left(x_{0}, y_{0}\right)$ and the integrated two-form operators at $\left(x_{1}, y_{1}\right)$ and $\left(x_{2}, y_{2}\right)$. There are three integrated one form operators, $\psi^{(1)}$, which we choose to be located at $w_{1}, w_{2}$ and $w_{3}$, and two integrated one-form operators, $\Omega^{(1)}$, whose locations are taken to be at $z_{1}$ and $z_{2}$. Then the Lorentzians, which appear in the integrand, are

$$
\begin{aligned}
& \mathcal{L}_{y}\left(w_{1}-x_{0}, y_{0}\right) \mathcal{L}_{y}\left(z_{1}-x_{0}, y_{0}\right) \mathcal{L}_{y}\left(z_{2}-x_{0}, y_{0}\right) \\
\times & \mathcal{L}_{y}\left(w_{2}-x_{1}, y_{1}\right)\left[\mathcal{L}_{y}\left(z_{1}-x_{1}, y_{1}\right) \mathcal{L}_{x}\left(z_{2}-x_{1}, y_{1}\right)-\mathcal{L}_{y}\left(z_{2}-x_{1}, y_{1}\right) \mathcal{L}_{x}\left(z_{1}-x_{1}, y_{1}\right)\right] \\
\times & \mathcal{L}_{y}\left(w_{3}-x_{2}, y_{2}\right)\left[\mathcal{L}_{y}\left(z_{1}-x_{2}, y_{2}\right) \mathcal{L}_{x}\left(z_{2}-x_{2}, y_{2}\right)-\mathcal{L}_{y}\left(z_{2}-x_{2}, y_{2}\right) \mathcal{L}_{x}\left(z_{1}-x_{2}, y_{2}\right)\right]
\end{aligned}
$$

As $y_{0}$ approaches zero, the three Lorentzians in the first line of eq. (C.2) tend to be sharply peaked (with width $\sim y_{0}$ ) and become delta function in the limit, $y_{0} \rightarrow 0$. Note that this implies that for small values of $y_{0}$ the integrand is also sharply peaked at $z_{1}=z_{2}$. Thus we assume that $\left(z_{1}-z_{2}\right)$ is small and has a fixed sign specified by the path ordering. Focusing on the second line of eq. (C.2) and on carrying out the $x_{1}$ integration, we find that the answer approaches zero as $z_{1} \rightarrow z_{2}$ unless $y_{1} \rightarrow 0$. This behavior can be summarized as follows

$$
\begin{aligned}
& \int_{-\infty}^{\infty} d x_{1} \mathcal{L}_{y}\left(w_{2}-x_{1}, y_{1}\right) \\
& \times {\left[\mathcal{L}_{y}\left(z_{1}-x_{1}, y_{1}\right) \mathcal{L}_{x}\left(z_{2}-x_{1}, y_{1}\right)-\mathcal{L}_{y}\left(z_{2}-x_{1}, y_{1}\right) \mathcal{L}_{x}\left(z_{1}-x_{1}, y_{1}\right)\right] } \\
&=\pi \mathcal{L}_{y}\left(w_{2}-x_{1}, y_{1}\right) \mathcal{L}_{x}\left(z_{1}-z_{2}, y_{1}\right)+\mathcal{O}\left(z_{1}-z_{2}\right)
\end{aligned}
$$

Then in the limit $z_{1} \rightarrow z_{2}$ one has 17

$$
\mathcal{L}_{x}\left(z_{1}-z_{2}, y_{1}\right)=\frac{\pi}{2} \operatorname{Sign}\left(z_{1}-z_{2}\right) \delta\left(y_{1}\right)
$$

where the factor $\frac{1}{2}$ reflects the fact that $y_{1}$ runs over the half line. Thus, for $y_{0}$ small but non-zero we obtain that the above delta function, $\delta\left(y_{1}\right)$, gets replaced by an even

17 We do $\operatorname{not}$ set $\operatorname{Sign}(0)=0$ (as is often conventional) because our limits are always taken from one side. 
function sharply peaked at $y_{1}=0$ with width $\sim y_{0}$. The third line in eq. (C.2) gives a similar result on carrying out the $x_{2}$ integration. Therefore evaluating eq. (C.2) in the limit, $y_{0} \rightarrow 0$, yields two identical Sign functions, which square to 1 , and a collection of delta functions, which are easy to integrate.

It is clear that taking the limit, $y_{0} \rightarrow 0$, and carrying out the $x_{1}$ and $x_{2}$ integrations, the only contribution arises when all the boundary operators are close to one another. In other words, the only contribution is a contact term with each pair of bulk integrals contributing $\frac{\pi^{2}}{2}$. If there were no path ordering to deal with, then each boundary integral would contribute a factor, $\pi$, with the end result being $\frac{\pi^{9}}{4}$.

\section{C.2. Handling the path ordering}

The boundary integrals are path-ordered and we need to carry out the integral for each ordering separately. However, the fact that they reduce to contact terms in the limit $y_{0} \rightarrow 0$ does simplify the analysis. First, since the operators, $\psi$, are identical, we can choose to order the operators such that $z_{1} \leq z_{2} \leq z_{3}$. Now all that remains is to work out the orderings of the boundary preserving operators, $\Omega$.

Second, note that all path orderings give the same answer. Each such ordering, however, is multiplied by a different $u_{i}$-dependent factor. At third order cyclic symmetry implies that there are four different cyclic invariant combinations of the variables, $u_{i}$ :

$$
\begin{array}{ll}
h_{1}^{(3)}=u_{1}^{3}+u_{2}^{3}+u_{3}^{3}, & h_{2}^{(3)}=u_{1} u_{2}^{2}+u_{2} u_{3}^{2}+u_{3} u_{1}^{2} \\
h_{3}^{(3)}=u_{1}^{2} u_{2}+u_{2}^{2} u_{3}+u_{3}^{2} u_{1}, & h_{4}^{(3)}=u_{1} u_{2} u_{3} .
\end{array}
$$

It is easy to see that there is one ordering (up to cyclic invariance) that can give rise to $h_{1}^{(3)}, 3$ distinct orderings that give rise to $h_{2}^{(3)}$ (and $h_{3}^{(3)}$ ) and 6 distinct orderings that give rise to $h_{4}^{(3)}$. Combining these different contributions, we see that

$$
\mathcal{W}_{3} \propto\left(h_{1}+3 h_{2}+3 h_{3}+6 h_{4}\right)=\left(u_{1}+u_{2}+u_{3}\right)^{3}
$$

This shows that only the combination $u \equiv\left(u_{1}+u_{2}+u_{3}\right)$ can appear (at this order). Hence, we can choose $u_{1}=u_{2}=u_{3}=\frac{u}{3}$ in order to make $\underline{X}$ and $\underline{U}$ commute. Then, the path-ordering is trivial and the final result from the integral is $\frac{\pi^{9}}{4}$. 


\section{C.3. The general case}

The argument of the previous subsections applies also for the two graphs that appear in evaluating the superpotential term, $\mathcal{W}_{4}$. Again only the combination $u \equiv\left(u_{1}+u_{2}+u_{3}\right)$ appears and the integral yields $\frac{\pi^{12}}{8}$. The conjecture is that the appearance of the combination, $u$, is true to all orders. We also suspect that the

integrals for the superpotential terms, $\mathcal{W}_{n}$, are given by $\frac{\pi^{3 n}}{2^{n-1}}$, i.e. a factor, $\frac{\pi^{2}}{2}$, from each bulk integration and a factor, $\pi$, from each boundary integration. However, for the term, $\mathcal{W}_{5}$, one finds that not all the integrands of the contributing graphs simplify to pure contact terms in the limit, $y_{0} \rightarrow 0$. Further, the structure of the graphs that appear for higher superpotential terms, $\mathcal{W}_{n}$, are more complicated and we have not studied them.

\section{Appendix D. Computation of the open-string mirror map on the torus}

In this appendix we determine the open-string mirror maps, $\mathcal{N}_{u}(\tau)$ and $\mathcal{N}_{X}(\tau, u)$. The starting point of this computation is the mirror-map relation (7.13), which, as discussed in the main text, arises from comparing the effective superpotentials of the B- and A-model, $\mathcal{W}$ and $\hat{\mathcal{W}}$.

As both correlators, $\Delta_{111}(\tau, u)$ and $\mu_{1}(\tau, \hat{u}) \equiv \hat{\Delta}_{111}\left(\tau, \hat{u}-u_{0}\right)$, are odd functions in $u$ and $\hat{u}$ respectively, the third power of the mirror map function, $\mathcal{N}_{X}^{3}$, must be even in the modulus, $u$. Thus it enjoys the expansion

$$
\mathcal{N}_{X}^{3}(\tau, u)=\sum_{k=0}^{+\infty} c_{2 k}(\tau) u^{2 k},
$$

and we need to determine the coefficients, $c_{2 k}$, by the relation (7.13).

In order to systematically compute the coefficients, $c_{2 k}(\tau)$, which directly specify the mirror map, $\mathcal{N}_{X}(\tau, u)$, it is convenient to first rewrite the functions, $\mu_{\ell}(\tau, \hat{u})$, as 
power series in the A-model variable, $\hat{u}$. The functions, $\mu_{\ell}(\tau, \hat{u})$, defined by eq. (7.10), become $[32,45]^{18}$

$$
\mu_{\ell}(\tau, \hat{u})=e^{\frac{2}{3} i(\ell-1) \pi} \sum_{m \in \mathbb{Z}} q^{\frac{3}{2}\left(\frac{1-\ell}{3}+m-\frac{1}{2}\right)^{2}} e^{2 \pi i\left(\frac{1-\ell}{3}+m-\frac{1}{2}\right)\left(\hat{u}-\frac{1}{2}\right)} .
$$

Note that $\mu_{1}(\tau, \hat{u})$, is odd in $\hat{u}$, and we also define the odd and even parts of the functions, $\mu_{2}(\tau, \hat{u})$ and $\mu_{3}(\tau, \hat{u})$ :

$$
\begin{aligned}
& \mu_{2}^{\mathrm{o}}(\tau, \hat{u})=\frac{1}{2}\left(\mu_{2}(\tau, \hat{u})-\mu_{2}(\tau,-\hat{u})\right)=\frac{1}{2}\left(\mu_{3}(\tau, \hat{u})-\mu_{3}(\tau,-\hat{u})\right), \\
& \mu_{2}^{\mathrm{e}}(\tau, \hat{u})=\frac{1}{2}\left(\mu_{2}(\tau, \hat{u})+\mu_{2}(\tau,-\hat{u})\right)=-\frac{1}{2}\left(\mu_{3}(\tau, \hat{u})+\mu_{3}(\tau,-\hat{u})\right) .
\end{aligned}
$$

After a few steps of algebra we arrive at the power series in the variable, $\hat{u}$ :

$$
\begin{aligned}
& \mu_{1}(\tau, \hat{u})=\sum_{k=0}^{+\infty}\left(\frac{4 i \pi}{3}\right)^{k} \frac{\pi}{(2 k+1) !} g^{(k)}(\tau) \hat{u}^{2 k+1}, \\
& \mu_{2}^{\mathrm{o}}(\tau, \hat{u})=\sum_{k=0}^{+\infty}\left(\frac{4 i \pi}{3}\right)^{k} \frac{\pi}{(2 k+1) !} h_{\mathrm{o}}^{(k)}(\tau) \hat{u}^{2 k+1}, \\
& \mu_{2}^{\mathrm{e}}(\tau, \hat{u})=\sum_{k=0}^{+\infty}\left(\frac{4 i \pi}{3}\right)^{k} \frac{i}{(2 k) !} h_{\mathrm{e}}^{(k)}(\tau) \hat{u}^{2 k} .
\end{aligned}
$$

The coefficient functions, $g^{(k)}(\tau), h_{\mathrm{o}}^{(k)}(\tau)$ and $h_{\mathrm{e}}^{(k)}(\tau)$, are the $k^{\text {th }}$ derivatives of the functions, $g(\tau), h_{0}(\tau)$ and $h_{\mathrm{e}}(\tau)$, which turn out to be equal to

$$
g(\tau)=2 \eta^{3}(3 \tau), \quad h_{\mathrm{o}}(\tau)=-a(\tau) \eta^{3}(3 \tau), \quad h_{\mathrm{e}}(\tau)=\eta(\tau) .
$$

Now, we have assembled all the ingredients to compute the open-string mirror maps (7.9). At order $u^{0}$ we find from the correlator, $\left(\kappa_{123}-\kappa_{132}\right)$, in $\mathcal{W}_{0}$ with eq. (5.12)

$$
c_{0}(\tau)=\frac{3 i \mathcal{I}_{0}}{\eta(\tau)}=\frac{i}{\eta(\tau)}
$$

18 The definition of the open-string modulus, $\hat{u}$, in ref. [32] differs from our open-string variable, $\hat{u}$, by a factor of $1 / 3$. As discussed in ref. [45], the global structure of the openstring moduli space of the 'long' branes $L_{a}$ leads in our conventions to the natural periodicity $\hat{u} \sim \hat{u}+1 \sim \hat{u}+\tau$. 
whereas at order $u^{1}$ we determine with the correlator, $\kappa_{111}$, in $\mathcal{W}_{1}$, the open-string mirror map, $\mathcal{N}_{u}(\tau)$, recorded in eq. (7.14). There is a consistency check by computing the function, $\mathcal{N}_{u}(\tau)$, as well from the correlator, $\left(\kappa_{123}+\kappa_{132}\right)$, in $\mathcal{W}_{1}$. This confirms the result stated in eq. (7.14).

Since we have already unambiguously determined the open-string mirror map, $\mathcal{N}_{u}(\tau)$, the remaining task is to computed the coefficient functions, $c_{2 k}(\tau)$. This is achieved by comparing in eq. (7.13) the coefficients, $u^{2 k}$, of the correlator, $\left(\kappa_{123}-\right.$ $\left.\kappa_{132}\right)$, at each order, and we obtain the recursion relation in terms of the modular functions 6.12 , 19

$$
\sum_{k=0}^{n} i\left(3 \pi^{2}\right)^{3(n-k)} \frac{\eta^{(n-k)}(\tau)}{(2(n-k)) !} c_{2 k}(\tau)=\tilde{G}_{2 n}(\tau) .
$$

Here we used the expansion (D.4) for the even function, $\mu_{2}^{\mathrm{e}}$, and the expression (6.11) for the B-model superpotential terms, $\mathcal{W}_{2 k}$.

With the recursion formula (D.7) we are now able to determine the first few coefficient functions, $c_{2 k}(\tau)$ :

$$
\begin{aligned}
c_{0}(\tau)= & \frac{i}{\eta(\tau)}, \\
c_{2}(\tau)= & \frac{i}{\eta(\tau)}\left(-\frac{27 \pi^{6}}{2} \frac{\eta^{\prime}(\tau)}{\eta(\tau)}\right), \\
c_{4}(\tau)= & -\frac{i}{\eta(\tau)} \tilde{G}_{4}(\tau)+\frac{\left(3 \pi^{2}\right)^{6} i}{4}\left(\frac{\eta^{\prime}(\tau)^{2}}{\eta(\tau)^{3}}-\frac{\eta^{\prime \prime}(\tau)}{6 \eta(\tau)^{2}}\right), \\
& \ldots
\end{aligned}
$$

The structure, which arises from the first few coefficients, $c_{2 k}$, gives rise to the mirrormap function, $\mathcal{N}_{X}^{3}(\tau, u)$,

$$
\mathcal{N}_{X}^{3}(\tau, u)=\frac{i}{\eta(\tau)} e^{-\frac{27}{8} i \pi^{5} G_{2}(\tau) u^{2}+d_{4} G_{4}(\tau) u^{4}+d_{6} G_{6}(\tau) u^{6}+\cdots} .
$$

Here the coefficients, $d_{4}, d_{6}, \ldots$, are determined by the higher order coefficients, $c_{2 k}$. The functions, $G_{4}$ and $G_{6}$, are the Eisenstein functions of modular weight 4 and 6 , and the general expression for the functions, $\mathcal{N}_{X}^{3}(\tau, u)$, is series in terms of all modular

\footnotetext{
19 Recall that $\tilde{G}_{2}(\tau) \equiv 0$.
} 
functions. This form is dictated by comparing yet again the modular properties of the correlators, $\left(\kappa_{123}-\kappa_{132}\right)$, of the B-model to the A-model. We know that this correlator, as perturbatively computed in the B-model, is modular invariant. However, the function, $\mu_{2}^{\mathrm{e}}$, which is the corresponding correlator in the A-model, is not modular invariant. Hence according to the mirror-map relation (8.13) the product, $\mathcal{N}_{X}^{3} \mu_{2}^{\mathrm{e}}$, must also be modular invariant. This, however, is precisely achieved by the stated mirror-map function (D.9), which contains the compensating non-modular Eisenstein function, $G_{2}$.

Except for determining the normalization, $\mathcal{N}_{u}$, we have only taken into account the even terms in $u$ in the effective superpotentials, $\mathcal{W}$ and $\hat{\mathcal{W}}$. In other words, we have only used the information encoded in the correlators, $\left(\kappa_{123}-\kappa_{132}\right)$. But the correlators, $\left(\kappa_{123}+\kappa_{132}\right)$ and $\kappa_{111}$, appearing only at odd orders in $u$, do also both determine the coefficient functions, $c_{2 k}(\tau)$, recursively. Thus determining the mirrormap, $\mathcal{N}_{X}$, via the correlators, $\left(\kappa_{123}+\kappa_{132}\right)$ and $\kappa_{111}$, serves as a highly non-trivial consistency check for the determined coefficient functions, $c_{2 k}$, and for the general method of computing the potential, $\mathcal{W}$, perturbatively.

In particular we obtain at third order in $u$ two consistency conditions:

$$
\begin{aligned}
& 0=\pi \mathcal{N}_{u} c_{2} g+\frac{2 \pi^{2} i}{9} \mathcal{N}_{u}^{3} c_{0} g^{\prime}-\frac{27 \pi^{9}}{8 \sqrt{2}} a^{2} q_{\mathrm{f}}^{-3}, \\
& 0=\pi \mathcal{N}_{u} c_{2} h_{\mathrm{o}}+\frac{2 \pi^{2} i}{9} \mathcal{N}_{u}^{3} c_{0} h_{\mathrm{o}}^{\prime}+\frac{9 \pi^{9}}{4 \sqrt{2}}\left(1-\frac{1}{4} a^{3}\right) q_{\mathrm{f}}^{-3} .
\end{aligned}
$$

The first relation arises from the correlator, $\kappa_{111}$, whereas the second relation comes from the correlator, $\left(\kappa_{123}+\kappa_{132}\right)$. Both identities are fulfilled with the previously computed quantities, $c_{0}, c_{2}$ and $\mathcal{N}_{u}$.

Similarly the two relations at order $u^{5}$, which are again satisfied by the already recursively determined quantities, read:20

$$
\begin{aligned}
& 0=\pi \mathcal{N}_{u} c_{4} g+\frac{2 \pi^{2} i}{9} \mathcal{N}_{u}^{3} c_{2} g^{\prime}-\frac{2 \pi^{3}}{135} \mathcal{N}_{u}^{5} c_{0} g^{\prime \prime}-3 \mathcal{I}_{5}\left(-\frac{3}{8} a^{4}-3 a\right) q_{\mathrm{f}}^{-5} \\
& 0=\pi \mathcal{N}_{u} c_{4} h_{\mathrm{o}}+\frac{2 \pi^{2} i}{9} \mathcal{N}_{u}^{3} c_{2} h_{\mathrm{o}}^{\prime}-\frac{2 \pi^{3}}{135} \mathcal{N}_{u}^{5} c_{0} h_{\mathrm{o}}^{\prime \prime}+3 \mathcal{I}_{5}\left(\frac{1}{16} a^{5}-\frac{1}{2} a^{2}\right) q_{\mathrm{f}}^{-5}
\end{aligned}
$$

20 This consistency condition also constraints the coefficient, $\mathcal{I}_{5}$, to $\mathcal{I}_{5}=\frac{\mathcal{I}_{1}^{5}+180 \mathcal{I}_{0}^{3} \mathcal{I}_{1} \mathcal{I}_{4}}{15 \mathcal{I}_{0}^{4}}$. 


\section{References}

[1] D. Gepner, "Exactly Solvable String Compactifications On Manifolds Of SU(N) Holonomy," Phys. Lett. B 199, 380 (1987).

[2] D. Gepner, "Space-Time Supersymmetry In Compactified String Theory And Superconformal Models," Nucl. Phys. B 296, 757 (1988).

[3] K. Hori, A. Iqbal and C. Vafa, "D-branes and mirror symmetry," arXiv:hepth/0005247.

[4] A. Kapustin and Y. Li, "D-branes in Landau-Ginzburg models and algebraic geometry," JHEP 0312, 005 (2003) arXiv:hep-th/0210296.

[5] I. Brunner, M. Herbst, W. Lerche and B. Scheuner, "Landau-Ginzburg realization of open string TFT," arXiv:hep-th/0305133.

[6] A. Kapustin and Y. Li, "D-branes in topological minimal models: The LandauGinzburg approach," JHEP 0407, 045 (2004) arXiv:hep-th/0306001].

[7] C. I. Lazaroiu, "On the boundary coupling of topological Landau-Ginzburg models," JHEP 0505, 037 (2005) arXiv:hep-th/0312286.

[8] K. Hori and J. Walcher, "F-term equations near Gepner points," JHEP 0501, 008 (2005) arXiv:hep-th/0404196.

[9] E. Witten, "On the Landau-Ginzburg description of $\mathrm{N}=2$ minimal models," Int. J. Mod. Phys. A 9, 4783 (1994) [arXiv:hep-th/9304026].

[10] S. Govindarajan, T. Jayaraman and T. Sarkar, "Worldsheet approaches to Dbranes on supersymmetric cycles," Nucl. Phys. B 580, 519 (2000) arXiv:hepth/9907131].

[11] S. Govindarajan and T. Jayaraman, "Boundary fermions, coherent sheaves and D-branes on Calabi-Yau manifolds," Nucl. Phys. B 618, 50 (2001) arXiv:hepth/0104126].

[12] S. Hellerman, S. Kachru, A. E. Lawrence and J. McGreevy, "Linear sigma models for open strings," JHEP 0207, 002 (2002) arXiv:hep-th/0109069.

[13] M. Bershadsky, S. Cecotti, H. Ooguri and C. Vafa, "Kodaira-Spencer theory of gravity and exact results for quantum string amplitudes," Commun. Math. Phys. 165, 311 (1994) arXiv:hep-th/9309140. 
[14] I. Antoniadis, E. Gava, K. S. Narain and T. R. Taylor, "Superstring threshold corrections to Yukawa couplings," Nucl. Phys. B 407, 706 (1993) arXiv:hepth/9212045].

[15] H. Ooguri and C. Vafa, "Knot invariants and topological strings," Nucl. Phys. B 577, 419 (2000) arXiv:hep-th/9912123.

[16] C. Vafa, "Superstrings and topological strings at large N," J. Math. Phys. 42, 2798 (2001) arXiv:hep-th/0008142.

[17] H. Ooguri and C. Vafa, "Gravity induced C-deformation," Adv. Theor. Math. Phys. 7, 405 (2004) arXiv:hep-th/0303063.

[18] R. Dijkgraaf and C. Vafa, "Matrix models, topological strings, and supersymmetric gauge theories," Nucl. Phys. B 644, 3 (2002) arXiv:hep-th/0206255.

[19] R. Dijkgraaf and C. Vafa, "On geometry and matrix models," Nucl. Phys. B 644, 21 (2002) arXiv:hep-th/0207106.

[20] A. Siqveland, "The Method of Computing Formal Moduli," J. Alg. 241, 292-327 (2001).

[21] S. K. Ashok, E. Dell'Aquila, D. E. Diaconescu and B. Florea, "Obstructed Dbranes in Landau-Ginzburg orbifolds," Adv. Theor. Math. Phys. 8, 427 (2004) arXiv:hep-th/0404167.

[22] J. Knapp and H. Omer, "Matrix factorizations, minimal models and Massey products," JHEP 0605, 064 (2006) arXiv:hep-th/0604189.

[23] A. Siqveland, "Global Matric Massey Products and the Compactified Jacobian of the E6-Singularity," J. Alg. 241 259-291 (2001).

[24] A. Siqveland, "Generalized Matric Massey Products for Graded Modules," arXiv:math.AG/0603425.

[25] M. R. Douglas, S. Govindarajan, T. Jayaraman and A. Tomasiello, "D-branes on Calabi-Yau manifolds and superpotentials," Commun. Math. Phys. 248, 85 (2004) arXiv:hep-th/0203173.

[26] B. Ezhuthachan, S. Govindarajan and T. Jayaraman, "A quantum McKay correspondence for fractional 2p-branes on LG orbifolds," JHEP 0508, 050 (2005) arXiv:hep-th/0504164.

[27] B. Ezhuthachan, S. Govindarajan and T. Jayaraman, "Fractional two-branes, toric orbifolds and the quantum McKay correspondence," arXiv:hep-th/0606154. 
[28] M. Herbst, C. I. Lazaroiu and W. Lerche, "Superpotentials, A(infinity) relations and WDVV equations for open topological strings," JHEP 0502, 071 (2005) arXiv:hep-th/0402110.

[29] M. Herbst, C. I. Lazaroiu and W. Lerche, "D-brane effective action and tachyon condensation in topological minimal models," JHEP 0503, 078 (2005) arXiv:hepth/0405138].

[30] A. Polishchuk and E. Zaslow, "Categorical mirror symmetry: The Elliptic curve," Adv. Theor. Math. Phys. 2, 443 (1998) [arXiv:math.ag/9801119].

[31] D. Cremades, L. E. Ibáñez and F. Marchesano, "Yukawa couplings in intersecting D-brane models," JHEP 0307, 038 (2003) arXiv:hep-th/0302105.

[32] I. Brunner, M. Herbst, W. Lerche and J. Walcher, "Matrix factorizations and mirror symmetry: The cubic curve," arXiv:hep-th/0408243.

[33] M. Herbst, W. Lerche and D. Nemeschansky, "Instanton geometry and quantum A(infinity) structure on the elliptic curve," arXiv:hep-th/0603085.

[34] E. Witten, "Mirror manifolds and topological field theory," arXiv:hep-th/9112056.

[35] B. R. Greene, C. Vafa and N. P. Warner, "Calabi-Yau manifolds and renormalization group flows," Nucl. Phys. B 324, 371 (1989).

[36] E. J. Martinec, "Algebraic geometry and effective Lagrangians," Phys. Lett. B 217, 431 (1989).

[37] E. Witten, "Phases of $\mathrm{N}=2$ theories in two dimensions," Nucl. Phys. B 403, 159 (1993) arXiv:hep-th/9301042.

[38] C. Vafa, "Topological Landau-Ginzburg models," Mod. Phys. Lett. A 6, 337 (1991).

[39] M. Herbst and C. I. Lazaroiu, "Localization and traces in open-closed topological Landau-Ginzburg models," JHEP 0505, 044 (2005) arXiv:hep-th/0404184.

[40] S. Kachru and E. Witten, "Computing the complete massless spectrum of a Landau-Ginzburg orbifold," Nucl. Phys. B 407, 637 (1993) arXiv:hepth/9307038].

[41] R. Dijkgraaf, H. L. Verlinde and E. P. Verlinde, "Topological Strings in $d<1$," Nucl. Phys. B 352, 59 (1991).

[42] W. Lerche, D. Lüst and N. P. Warner, "Duality Symmetries In N=2 LandauGinzburg Models," Phys. Lett. B 231, 417 (1989). 
[43] W. Lerche, D. J. Smit and N. P. Warner, "Differential equations for periods and flat coordinates in two-dimensional topological matter theories," Nucl. Phys. B 372, 87 (1992) arXiv:hep-th/9108013.

[44] N. P. Warner, "Supersymmetry in boundary integrable models," Nucl. Phys. B 450, 663 (1995) arXiv:hep-th/9506064.

[45] S. Govindarajan, H. Jockers, W. Lerche and N. P. Warner, "Tachyon condensation on the elliptic curve," arXiv:hep-th/0512208.

[46] S. K. Ashok, E. Dell'Aquila and D. E. Diaconescu, "Fractional branes in Landau-Ginzburg orbifolds," Adv. Theor. Math. Phys. 8, 461 (2004) arXiv:hepth/0401135.

[47] M. R. Douglas, B. Fiol and C. Römelsberger, "The spectrum of BPS branes on a noncompact Calabi-Yau," JHEP 0509, 057 (2005) arXiv:hep-th/0003263.

[48] J. Walcher, "Stability of Landau-Ginzburg branes," J. Math. Phys. 46, 082305 (2005) arXiv:hep-th/0412274.

[49] E. Witten, "On background independent open string field theory," Phys. Rev. D 46, 5467 (1992) arXiv:hep-th/9208027.

[50] S. L. Shatashvili, "Comment on the background independent open string theory," Phys. Lett. B 311, 83 (1993) arXiv:hep-th/9303143.

[51] D. Kutasov, M. Mariño and G. W. Moore, "Remarks on tachyon condensation in superstring field theory," arXiv:hep-th/0010108.

[52] V. Niarchos and N. Prezas, "Boundary superstring field theory," Nucl. Phys. B 619, 51 (2001) arXiv:hep-th/0103102.

[53] I. Brunner, M. R. Douglas, A. E. Lawrence and C. Römelsberger, "D-branes on the quintic," JHEP 0008, 015 (2000) arXiv:hep-th/9906200.

[54] S. Kachru, S. Katz, A. E. Lawrence and J. McGreevy, "Open string instantons and superpotentials," Phys. Rev. D 62, 026001 (2000) arXiv:hep-th/9912151].

[55] P. S. Aspinwall and S. Katz, "Computation Of Superpotentials For D-Branes," Commun. Math. Phys. 264, 227 (2006) arXiv:hep-th/0412209.

[56] C. Hofman and W. K. Ma, "Deformations of topological open strings," JHEP 0101, 035 (2001) arXiv:hep-th/0006120.

[57] M. A. A. van Leeuwen, A. M. Cohen and B. Lisser, "LiE 2.2.2: A computer algebra package," http://www-math.univ-poitiers.fr/〜maavl/LiE/. 
[58] S. Kachru, S. Katz, A. E. Lawrence and J. McGreevy, "Mirror symmetry for open strings," Phys. Rev. D 62, 126005 (2000) arXiv:hep-th/0006047].

[59] M. Herbst, "Quantum A-infinity structures for open-closed topological strings," arXiv:hep-th/0602018.

[60] A. N. Schellekens and N. P. Warner, "Anomalies, Characters And Strings," Nucl. Phys. B 287, 317 (1987).

[61] E. Witten, "Quantum background independence in string theory," arXiv:hepth/9306122. 